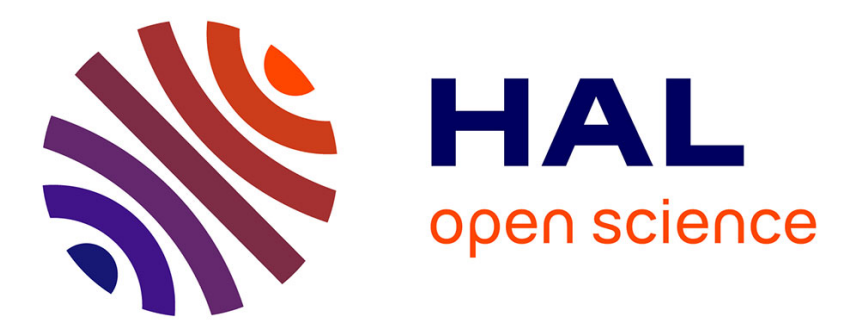

\title{
Advanced spectral anisotropic modelling for shear flows
} Antoine Briard, Benoît-Joseph Gréa, Vincent Mons, Claude Cambon, Thomas Gomez, Pierre Sagaut

\section{To cite this version:}

Antoine Briard, Benoît-Joseph Gréa, Vincent Mons, Claude Cambon, Thomas Gomez, et al.. Advanced spectral anisotropic modelling for shear flows. Journal of Turbulence, 2018, 19 (7), pp.570-599. 10.1080/14685248.2018.1478092 . hal-02112204

\section{HAL Id: hal-02112204 https://hal-amu.archives-ouvertes.fr/hal-02112204}

Submitted on 26 Apr 2019

HAL is a multi-disciplinary open access archive for the deposit and dissemination of scientific research documents, whether they are published or not. The documents may come from teaching and research institutions in France or abroad, or from public or private research centers.
L'archive ouverte pluridisciplinaire HAL, est destinée au dépôt et à la diffusion de documents scientifiques de niveau recherche, publiés ou non, émanant des établissements d'enseignement et de recherche français ou étrangers, des laboratoires publics ou privés. 


\title{
Advanced spectral anisotropic modelling for shear flows
}

\author{
Antoine Briard a,b , Benoît-Joseph Gréac ${ }^{c}$ Vincent Mons ${ }^{d}$, Claude Cambon $^{\mathrm{e}}$, \\ Thomas Gomez $z^{f}$ and Pierre Sagaut ${ }^{b}$ \\ ${ }^{a}$ CMLA, ENS Cachan, CNRS, Université Paris-Saclay, Cachan, France; ${ }^{b}$ CNRS Centrale Marseille, M2P2 \\ UMR7340, Aix-Marseille Université, Marseille, France; ${ }^{\mathrm{C} D I F}, \mathrm{DAM}, \mathrm{CEA}$, Arpajon, France; ${ }^{\mathrm{d}}$ Department of \\ Mechanical Engineering, Johns Hopkins University, Baltimore, MD, USA; ' Laboratoire de Mécanique des \\ Fluides et d'Acoustique, Ecole Centrale de Lyon, Lyon, France; f Univ. Lille, Onera, CNRS, Centrale Lille, Arts et \\ Metiers ParisTech, FRE 2017 - LMFL - Laboratoire de Mécanique des Fluides de Lille Kampé de Fériet, F-59000 \\ Lille, France
}

\begin{abstract}
In this work, the spectral modelling developed in MCS [Mons, Cambon, Sagaut. A spectral model for homogeneous shear-driven anisotropic turbulence in terms of spherically-averaged descriptors. J Fluid Mech. 2016;788:147-182] for shear-driven turbulence is further analysed and then improved. First, using self-similarity arguments, it is shown that the asymptotic kinetic energy exponential growth rate $\gamma$ is independent of the large scales infrared slope $\sigma$, with $E(k \rightarrow$ $0) \sim k^{\sigma}$, unlike unstably stratified homogeneous turbulence where $\gamma$ strongly depends on $\sigma$. The MCS model relies on the truncation at the second order of the spectral two-point velocity correlation expansion into spherical harmonics. The expansion is here pursued at the next even order, the fourth one: the noteworthy consequence is that $\gamma$ is decreased compared to MCS and is thus closer to values obtained in direct numerical simulations and experiments. Finally, some analytical considerations about odd-order contributions in the expansion of polarisation anisotropy are proposed.
\end{abstract}

\section{Introduction}

Natural turbulent flows represent a great challenge in the turbulence community in terms of modelling. Indeed, in atmospheric turbulent flows, for example, several complex mechanisms strongly interact in an intricate manner, on distinct characteristic time scales, such as rotation, stratification, helicity, and shear. Among these mechanisms, shear flows are probably the most complex, since no symmetry at all survive, unlike rotation and stratification which nevertheless remain statistically axisymmetric.

In the past years, the authors have analysed separately each of these configurations with the help of an adapted eddy-damped quasi-normal Markovian (EDQNM) closure able of handling strongly anisotropic flows, in order to better understand what are the intrinsic properties of each mechanism. A significant amount of results, both theoretical 
and numerical, were exposed in various publications [1-9], and still a lot of work needs to be done regarding shear flows specifically. Indeed, the anisotropic EDQNM model developed in Mons, Cambon, and Sagaut (MCS) [1] handles very satisfactorily various straining processes, for instance when the mean-velocity gradient matrix is symmetric, like in axisymmetric contractions or expansions, or in plane distortions. However, for pure plane shear flows where this matrix is not symmetric and has only one non-zero component, the MCS model gives a kinetic energy exponential growth rate $\gamma$ larger than common values obtained in direct numerical simulations (DNS): see [3] for a review. Despite the clear tendency of increasing values of $\gamma$ in more recent DNS [10,11] which can reach larger Reynolds numbers with a better spatial resolution, a discrepancy still remains. This quantitative issue regarding the value of $\gamma$ is probably due to a lack of angular information regarding the distribution of anisotropy within our anisotropic EDQNM modelling. Consequently, we focus here on pure plane shear flows, a particular case of shear-driven turbulence. The reader should nevertheless keep in mind that the model is more general and can handle various shear-driven configurations.

In homogeneous incompressible turbulence, the spectral velocity-velocity correlation $\hat{R}_{i j}$ can be exactly decomposed into directional and polarisation anisotropies [12,13], which are quantified by the two scalar quantities $\mathcal{E}(\boldsymbol{k}, t)$ and $Z(\boldsymbol{k}, t)$, which both depend on time and on the wavevector $\boldsymbol{k}$. In order to deal with analytical expressions in the modelling of anisotropy, $\mathcal{E}$ and $Z$ are expanded into spherical harmonics, and this expansion is further truncated at the first non-trivial order, the second one, the zeroth-order being the isotropic state where $Z=0$ and $\mathcal{E}=E /\left(4 \pi k^{2}\right)$, with $E$ being the kinetic energy spectrum. To further reduce the numerical cost of the simulations, the detailed equations for the directional and polarisation anisotropies are spherically averaged.

The numerical results obtained with such an approximation were thoroughly discussed in the previously mentioned references and compared quantitatively well with both DNS and experiments, in various configurations, from axisymmetric contractions, expansions, and plane distortions $[1,3]$, to the transport of a passive scalar field in the presence of a mean gradient with a variable Prandtl number [2,14], along with the case of unstably stratified turbulence [4]. However, for shear flows, the model could not recover accurately the anisotropy distribution by investigating, for instance, several components of the global indicator $b_{i j}=\left\langle u_{i} u_{j}\right\rangle / 2 K-\delta_{i j} / 3$, where $K=\int_{0}^{\infty} E(k) \mathrm{d} k$ is the kinetic energy, nor the asymptotic value of the kinetic exponential growth rate $\gamma$ as said earlier. Despite these defects, a good agreement was found for the shear parameter $S^{*}=2 \mathrm{KS} / \epsilon$, where $S$ is the shear rate and $\epsilon$ the kinetic energy dissipation rate, an important quantity for one-point modelling [3]. Thus, it was concluded that anisotropy was not well-enough captured, and more precisely polarisation anisotropy [15], with notably a too large value for $b_{13}$ (for $\left.S=-\partial U_{1} / \partial x_{3}\right)$. So far, it was impossible to quantify the anisotropic information lost by the second-order truncation. Therefore, we propose here to go beyond the second-order truncation and deal analytically with the fourth-order, to know for sure if this allows to decrease the exponential growth rate $\gamma$ or not. The derivation itself is rather complex and constitutes one of the main results of the present work. The second significant finding is an analytical proof showing an essential difference between the dynamics of shear flows and unstably stratified turbulence, configurations both analysed with the anisotropic EDQNM model, and in which the kinetic energy grows exponentially, but with completely different properties. 
It is worth recalling that the present model addresses only second-order moments of the velocity field, so that high-order statistics are not available. It notably implies that we cannot investigate intermittent properties of shear flows [16]. However, intermittent statistics are often observed for high-order moments of the velocity field [16-19] so that it remains compatible with our approach. Indeed, it has been observed with the model in $[1,3]$ that small scales of the velocity field second-order moments return to isotropy at large Reynolds numbers, a feature consistent with DNS and experiments [20-22]: nevertheless, some perspectives to study higher-order statistics with similar modelling techniques are discussed in the concluding section.

Finally, let us mention that other tools to analyse anisotropic features of shear flows are possible. For example, our approach is consistent with the irreducible representation of the $\mathrm{SO}(3)$ symmetry group that yields expansions in terms of spherical harmonics as well [23]. Applications to shear flows are also developed directly in physical space, with focus on anisotropic structure functions (see [24] for a review): note that expansions in terms of the separation vector $\boldsymbol{r}$ in physical space and in terms of $\boldsymbol{k}$ in spectral space can be partly reconciled [8]. In a recent work [25], the basis functions of the $\mathrm{SO}(3)$ symmetry group were used to derive an analytical dynamical model in the rapid distortion linear limit, instead of analysing statistics resulting from DNS as usual. This is in the spirit of the present approach where evolution equations, fully non-linear, however, are written for tensors corresponding to these basis functions.

The paper is organised as follows: as a starting point, the MCS model with the secondorder truncation of the spherical harmonics expansion is recalled in Section 2, with some details gathered in Appendix 1. A first new result is presented regarding the independence of the exponential growth rate with the large scales initial conditions. Then, the fourthorder modelling is presented in Section 3 along with the corresponding new production and non-linear terms. Finally, in Section 4, some analytical developments about odd-order terms of the expansion for the polarisation $Z$ are presented, unfortunately inconclusive. Discussions and further perspectives are gathered in Section 5.

\section{The anisotropic EDQNM modelling for homogeneous turbulence}

In this part, the main elements of the MCS anisotropic spectral model are recalled for clarity. First, the so-called $\mathcal{E}-Z$ decomposition of the spectral two-point second-order velocity-velocity correlation is presented. Afterwards, the modelling of anisotropy through spherically averaged deviatoric descriptors is exposed, along with brief details about the EDQNM procedure used to close the non-linear terms in the evolution equations. Then, the final spherically averaged equations for homogeneous turbulence are derived.

\subsection{The $\mathcal{E}-Z$ decomposition}

The spectral two-point second-order velocity-velocity correlation, or spectral Reynolds tensor, is defined as

$$
\hat{R}_{i j}(\boldsymbol{k}, t) \delta(\boldsymbol{k}-\boldsymbol{p})=\left\langle\hat{u}_{i}^{*}(\boldsymbol{p}, t) \hat{u}_{j}(\boldsymbol{k}, t)\right\rangle,
$$

where $\hat{\imath}$ denotes the Fourier transform, $(\cdot)^{*}$ is the complex conjugate, $\langle\cdot\rangle$ is an ensemble average, and $\boldsymbol{k}$ and $\boldsymbol{p}$ are wavevectors. The tensor $\hat{R}_{i j}$ further verifies the so-called Craya 
equation, namely

$$
\left(\frac{\partial}{\partial t}-A_{l n} k_{l} \frac{\partial}{\partial k_{n}}+2 v k^{2}\right) \hat{R}_{i j}(\boldsymbol{k})+M_{i n}(\boldsymbol{k}) \hat{R}_{n j}(\boldsymbol{k})+M_{j n}(\boldsymbol{k}) \hat{R}_{n i}(\boldsymbol{k})=T_{i j}^{\mathrm{NL}}(\boldsymbol{k}),
$$

where $v$ is the kinematic viscosity, $T_{i j}^{\mathrm{NL}}$ is the total non-linear transfer, $M_{i j}(\boldsymbol{k})=\left(\delta_{i n}-\right.$ $\left.2 \alpha_{i} \alpha_{n}\right) A_{n j}$ with $\alpha_{i}=k_{i} / k$, and $A_{l n}=\partial U_{l} / \partial x_{n}$ the mean-velocity gradient matrix, whose symmetric and antisymmetric parts are respectively identified with + and - superscripts in what follows.

Since $\hat{R}_{i j}$ is a homogeneous, symmetric, and solenoidal second-order tensor, it can be intrinsically and exactly decomposed into three distinct parts [12]

$$
\begin{gathered}
\hat{R}_{i j}(\boldsymbol{k})=\hat{R}_{i j}^{(\mathrm{iso})}(\boldsymbol{k})+\hat{R}_{i j}^{(\mathrm{dir})}(\boldsymbol{k})+\hat{R}_{i j}^{(\mathrm{pol})}(\boldsymbol{k}), \\
=\frac{E(k)}{4 \pi k^{2}} P_{i j}(\boldsymbol{k})+\left(\mathcal{E}(\boldsymbol{k})-\frac{E(k)}{4 \pi k^{2}}\right) P_{i j}(\boldsymbol{k})+\Re\left(Z(\boldsymbol{k}) N_{i}(\boldsymbol{k}) N_{j}(\boldsymbol{k})\right),
\end{gathered}
$$

where $P_{i j}=\delta_{i j}-\alpha_{i} \alpha_{j}$ is a projector and $N_{i}$ is a helical mode $[13,26]$, perpendicular to the wavevector $\boldsymbol{k}$. These three parts in the decomposition (4) refer to the isotropic contribution, the directional anisotropy, which is the difference between the energy along the wavevector $\boldsymbol{k}$ and the spherically averaged energy, and the polarisation anisotropy, which reflects the difference of energy between components of $\hat{R}_{i j}$. The kinetic energy density $\mathcal{E}(\boldsymbol{k}, t)$ is linked to directional anisotropy and is connected to the kinetic energy spectrum $E(k, t)$ through a spherical integration on a sphere $S_{k}$ of radius $k$

$$
E(k, t)=\int_{S_{k}} \mathcal{E}(\boldsymbol{k}, t) \mathrm{d}^{2} \boldsymbol{k}=\int_{S_{k}} \frac{\hat{R}_{i i}(\boldsymbol{k}, t)}{2} \mathrm{~d}^{2} \boldsymbol{k},
$$

whereas the complex-valued scalar $Z(\boldsymbol{k}, t)$ reflects polarisation anisotropy and reads

$$
Z(\boldsymbol{k}, t)=\frac{\hat{R}_{i j}(\boldsymbol{k}, t)}{2} N_{i}(-\boldsymbol{k}) N_{j}(-\boldsymbol{k}) .
$$

Both $\mathcal{E}$ and $Z$ verify exact evolution equations which can be found in several references $[1,8]$, namely

$$
\begin{gathered}
\left(\frac{\partial}{\partial t}-A_{\ln } k_{l} \frac{\partial}{\partial k_{n}}+2 \nu k^{2}\right) \mathcal{E}-A_{i j}^{+} \alpha_{i} \alpha_{j} \mathcal{E}+A_{i j}^{+} \Re\left(Z N_{i} N_{j}\right)=T_{\mathcal{E}}, \\
\left(\frac{\partial}{\partial t}-A_{\ln } k_{l} \frac{\partial}{\partial k_{n}}+2 \nu k^{2}\right) Z+2 \mathrm{i} Z \Omega_{\mathrm{CH}}+A_{i j}^{+} N_{i}^{*} N_{j}^{*} \mathcal{E}+A_{i j} N_{i}^{*} N_{j} Z=T_{Z},
\end{gathered}
$$

where $\Omega_{\mathrm{CH}}$ reflects the motion of the time-evolving Craya-Herring frame with respect to a fixed reference [1,27], and with $T_{\mathcal{E}}=T_{i i}^{\mathrm{NL}} / 2$ and $T_{Z}=T_{i j}^{\mathrm{NL}} N_{i}^{*} N_{j}^{*} / 2$. One could solve these exact evolution equations, but this would be extremely demanding in terms of computational resources. Note that considering the variables $(k \mathcal{E})$ and $(k Z)$ instead of only $\mathcal{E}$ and $Z$ simplifies the equations, as done in [7]. 
Instead, we choose to model anisotropy in two steps: first, the non-linear directional and polarisation transfers $T_{\mathcal{E}}$ and $T_{Z}$ are closed by a classical EDQNM procedure. Afterwards, both $\mathcal{E}$ and $Z$ are expanded into spherical harmonics to obtain explicit expressions for the directional and polarisation parts of the spectral Reynolds tensor. This expansion was further truncated at the second order for the sake of simplicity in [1]: the immediate drawback is the loss of angular anisotropic information which makes the production terms, linear with the mean-velocity gradient matrix $A_{l n}$, not exact anymore. The consequences of such an approximation were thoroughly examined and discussed in several references [1-3], so that they are not further argued here. The aim of this study is precisely to improve the modelling of anisotropy through a truncation at the next even order, the fourth-one, in Section 3. Before that, for consistency and clarity, the details of the truncation at the secondorder are recalled in the following part: in addition, a new theoretical feature is presented to better understand the exponential growth rate of kinetic energy obtained numerically with the model.

\subsection{Truncated expansion of $\mathcal{E}$ and $Z$ at the second-order}

The expansion into spherical harmonics of $\mathcal{E}$ and $Z$ is the key ingredient for the modelling of anisotropy. Such expansions read

$$
\begin{gathered}
\mathcal{E}(\boldsymbol{k})=\mathcal{E}_{0}(k)\left(1+U_{i j}^{(\mathrm{dir}) 2}(k) \alpha_{i} \alpha_{j}+U_{i j p q}^{(\mathrm{dir}) 4}(k) \alpha_{i} \alpha_{j} \alpha_{p} \alpha_{q}+\cdots\right), \\
Z(\boldsymbol{k})=\frac{1}{2} \mathcal{E}_{0}(k)\left(U_{i j}^{(\mathrm{pol}) 2}(k)+U_{i j p q}^{(\mathrm{pol}) 4}(k) \alpha_{p} \alpha_{q}+\cdots\right) N_{i}^{*}(\boldsymbol{k}) N_{j}^{*}(\boldsymbol{k}),
\end{gathered}
$$

with $\mathcal{E}_{0}=E /\left(4 \pi k^{2}\right)$, and where time dependence has been omitted for clarity. The determination of the dynamics of the symmetric and deviatoric second-order tensors $U_{i j}^{(\mathrm{dir}) 2}$ and $U_{i j}^{(\mathrm{pol}) 2}$ was the topic of [1], whereas the determination of that of the fourth-order tensors $U_{i j p q}^{(\mathrm{dir}) 4}$ and $U_{i j p q}^{(\mathrm{pol}) 4}$ constitutes one of the two theoretical contributions of this work, addressed in detail in Section 3. Odd-order terms of the expansion of $\mathcal{E}$ are exactly zero, which comes from the symmetry of $\hat{R}_{i j}[12,23]$; the zeroth-order corresponds to isotropy, configuration in which the polarisation anisotropy $Z=0$. The possibility of non-zero odd-order terms for $Z$ is discussed later on in Section 4.

As mentioned before, the spherical average transforms the $\boldsymbol{k}$-dependence of the twopoint second-order correlations into a $k$ one and thus strongly reduces the computational time of the simulations but also causes a loss of information regarding the distribution of anisotropy of the flow. In [1], part of this information was nevertheless recovered, thanks to the deviatoric and symmetric tensors $H_{i j}^{(\mathrm{dir})}$ and $H_{i j}^{(\mathrm{pol})}$, which can be linked to $U_{i j}^{(\mathrm{dir}) 2}$ and $U_{i j}^{(\mathrm{pol}) 2}$ through

$$
\begin{gathered}
2 E(k, t) H_{i j}^{(\mathrm{dir})}(k, t)=\int_{S_{k}} \hat{R}_{i j}^{(\mathrm{dir})}(\boldsymbol{k}, t) \mathrm{d}^{2} \boldsymbol{k}=-\frac{2}{15} U_{i j}^{(\mathrm{dir}) 2}(k, t) E(k, t), \\
2 E(k, t) H_{i j}^{(\mathrm{pol})}(k, t)=\int_{S_{k}} \hat{R}_{i j}^{(\mathrm{pol})}(\boldsymbol{k}, t) \mathrm{d}^{2} \boldsymbol{k}=\frac{2}{5} U_{i j}^{(\mathrm{pol}) 2}(k, t) E(k, t) .
\end{gathered}
$$


Hence, the second-order spectral tensor is given by

$$
\phi_{i j}(k, t)=\int_{S_{k}} \hat{R}_{i j}(\boldsymbol{k}, t) \mathrm{d}^{2} \boldsymbol{k}=2 E(k, t)\left(\frac{\delta_{i j}}{3}+H_{i j}^{(\mathrm{dir})}(k, t)+H_{i j}^{(\mathrm{pol})}(k, t)\right) .
$$

With these expressions (11) and (12), it is thus possible to determine the closed expressions of $\mathcal{E}$ and $Z$ at the second-order, namely

$$
\mathcal{E}(\boldsymbol{k})=\mathcal{E}_{0}(k)\left(1-15 H_{i j}^{(\mathrm{dir})}(k) \alpha_{i} \alpha_{j}\right), \quad Z(\boldsymbol{k})=\frac{5}{2} \mathcal{E}_{0}(k) H_{i j}^{(\mathrm{pol})}(k) N_{i}^{*}(\boldsymbol{k}) N_{j}^{*}(\boldsymbol{k}) .
$$

For now, only the truncated expansions at the second-order are considered, so that the modelled spectral Reynolds tensor reads

$$
\hat{R}_{i j}=\underbrace{\mathcal{E}_{0} P_{i j}\left(1-15 H_{p q}^{(\mathrm{dir})} \alpha_{p} \alpha_{q}\right)}_{\hat{R}_{i j}^{\mathrm{e} 2}}+\underbrace{5 \mathcal{E}_{0}\left(P_{i n} P_{j m} H_{m n}^{(\mathrm{pol})}+\frac{1}{2} P_{i j} H_{p q}^{(\mathrm{pol})} \alpha_{p} \alpha_{q}\right)}_{\hat{R}_{i j}^{z 2}} .
$$

In the following section, the evolution equations of the three main spectra $E, E H_{i j}^{(\mathrm{dir})}$, and $E H_{i j}^{(\mathrm{pol})}$ are given within the anisotropic EDQNM framework.

\subsection{The EDQNM procedure and spherically averaged equations}

The final step of the anisotropic EDQNM modelling is to close the non-linear transfers $T_{\mathcal{E}}$ and $T_{Z}$ using a classical EDQNM procedure. Details about the fundamentals of EDQNM can be found, for instance, in [28] for HIT, or in [1] with the anisotropic formalism. The modelled equation (15) of $\hat{R}_{i j}$ is further injected into the quasi-normal expression of the non-linear transfers. The eddy-damping term is kept isotropic for generality purposes and also to avoid the introduction of new arbitrary constants: this has proven to be relevant (in non-rotating flows) in various comparisons performed against DNS and experiments in different configurations [1-4].

After some algebra, one obtains the closed expressions of the non-linear transfers $T_{\mathcal{E}}$ and $T_{Z}$ in the evolution equations (7) and (8) of $\mathcal{E}$ and $Z$

$$
\begin{aligned}
T_{\mathcal{E}}= & 2 \int \theta_{k p q} k p\left[\left(\mathcal{E}^{\prime \prime}+\Re X^{\prime \prime}\right)\left(\left(x y+z^{3}\right)\left(\mathcal{E}^{\prime}-\mathcal{E}\right)-z\left(1-z^{2}\right)\left(\Re X^{\prime}-\Re X\right)\right)\right. \\
& \left.+\Im X^{\prime \prime}\left(1-z^{2}\right)\left(x \Im X-y \Im X^{\prime}\right)\right] \mathrm{d}^{3} p, \\
T_{Z}= & 2 \int \theta_{k p q} k p \mathrm{e}^{-2 i \lambda}\left[( \mathcal { E } ^ { \prime \prime } + \Re X ^ { \prime \prime } ) \left(\left(x y+z^{3}\right)\left(\Re X^{\prime}-X\right)-z\left(1-z^{2}\right)\left(\mathcal{E}^{\prime}-\mathcal{E}\right)\right.\right. \\
& \left.\left.+i\left(y^{2}-z^{2}\right) \Im X^{\prime}\right)+i \Im X^{\prime \prime}\left(1-z^{2}\right)\left(x(\mathcal{E}+X)-\mathrm{i} y \Im X^{\prime}\right)\right] \mathrm{d}^{3} p,
\end{aligned}
$$

with $\mathcal{E}(\boldsymbol{k}, t)=\mathcal{E}, \mathcal{E}(\boldsymbol{p}, t)=\mathcal{E}^{\prime}, \mathcal{E}(\boldsymbol{q}, t)=\mathcal{E}^{\prime \prime}, X=Z(\boldsymbol{k}, t) \mathrm{e}^{2 \mathrm{i} \lambda}, X^{\prime}=Z(\boldsymbol{p}, t) \mathrm{e}^{2 \mathrm{i} \lambda^{\prime}}$, and $X^{\prime \prime}=$ $Z(\boldsymbol{q}, t) \mathrm{e}^{2 \mathrm{i} \lambda^{\prime \prime}}$. The $\lambda, \lambda^{\prime}$, and $\lambda^{\prime \prime}$ are rotation angles around $\boldsymbol{k}, \boldsymbol{p}$, and $\boldsymbol{q}$ which permit to perform the calculations in a frame more adapted than the Craya-Herring one [27]. $x, y$, and $z$ are the cosines of the angles formed by $\boldsymbol{p}$ and $\boldsymbol{q}, \boldsymbol{q}$ and $\boldsymbol{k}$, and $\boldsymbol{k}$ and $\boldsymbol{p}$, respectively. And 
$\theta_{k p q}$ is the characteristic time of the third-order correlations, defined more precisely in Appendix 1. Furthermore, quadratic contributions of anisotropy (terms like $H_{i l}^{(\mathrm{dir})} H_{j l}^{\text {(dir) }}$, $H_{i l}^{(\mathrm{dir})} H_{j l}^{(\mathrm{pol})}, \ldots$ ) were discarded in the non-linear transfers, because they were assumed to be negligible [1,3]: this assumption is successfully assessed for the first time in Section 2.5.

Consequently, after spherical averaging, one ends up with three generalised Lin equations that describe the evolution of the isotropic, directional and polarisation parts of the turbulent velocity field, given under the same compact expression

$$
\begin{gathered}
\left(\frac{\partial}{\partial t}+2 v k^{2}\right) E(k, t)=S^{\mathrm{L}(\text { iso })}(k, t)+S^{\mathrm{NL}(\text { iso })}(k, t), \\
\left(\frac{\partial}{\partial t}+2 v k^{2}\right) E(k, t) H_{i j}^{(\mathrm{dir})}(k, t)=S_{i j}^{\mathrm{L}(\mathrm{dir})}(k, t)+S_{i j}^{\mathrm{NL}(\mathrm{dir})}(k, t), \\
\left(\frac{\partial}{\partial t}+2 v k^{2}\right) E(k, t) H_{i j}^{(\text {pol })}(k, t)=S_{i j}^{\mathrm{L}(\text { pol })}(k)+S_{i j}^{\mathrm{NL}(\text { pol })}(k, t),
\end{gathered}
$$

where $S^{\mathrm{NL}(\mathrm{iso})}, S_{i j}^{\mathrm{NL}(\mathrm{dir})}$, and $S_{i j}^{\mathrm{NL}(\mathrm{pol})}$ are the non-linear spherically averaged transfers, and $S^{\mathrm{L}(\mathrm{iso})}, S_{i j}^{\mathrm{L}(\mathrm{dir})}$, and $S_{i j}^{\mathrm{L}(\mathrm{pol})}$ are the production terms resulting from the presence of a meanvelocity gradient. The explicit expressions of these linear and non-linear transfers are given in Appendix 1.

Thanks to the previous spectral anisotropy descriptors $H_{i j}^{(\mathrm{dir})}$ and $H_{i j}^{(\mathrm{pol})}$, one can express the deviatoric normalised part of the Reynolds stress tensor, which refers to the global anisotropy of the flow, according to

$$
b_{i j}(t)=\frac{\left\langle u_{i} u_{j}\right\rangle}{2 K}-\frac{\delta_{i j}}{3}=\frac{1}{K(t)} \int_{0}^{\infty} E(k, t)\left[H_{i j}^{(\mathrm{dir})}(k, t)+H_{i j}^{(\mathrm{pol})}(k, t)\right] \mathrm{d} k .
$$

The three generalised Lin equations (18), (19), and (20) constitute the main result of MCS. This spectral modelling was then applied in [3] exclusively for shear flows. Notably, it was shown in the latter reference that:

- The kinetic energy grows exponentially at large $S t$ and Reynolds numbers at the rate $0.33 \leq \gamma \leq 0.34$, with $K \sim \exp (\gamma S t)$.

- This exponential growth rate $\gamma$ is found numerically to be independent of the large scales initial conditions $\sigma$, where $E\left(k<k_{L}\right) \sim k^{\sigma}$, with $k_{L}$ being the integral wavenumber [29]. Such an independence to large scales was also reported in [30] for shear flows.

- This value $\gamma \simeq 0.33$ is higher than common values obtained in DNS and experiments, as reported in Table 1 of [3], even though more recent numerical studies report larger exponential growth rates.

In the following part, we come back on both this value for $\gamma$ and on its independence with regard to $\sigma$ with theoretical arguments. In addition, a qualitative comparison is made with the asymptotic regimes of unstably stratified homogeneous turbulence, where the kinetic energy also grows exponentially but with a strong dependence on $\sigma$. 


\subsection{The exponential growth rate $\gamma$ of kinetic energy}

In this section, we provide theoretical arguments to explain both the rather large value of the kinetic energy exponential growth rate $\gamma=0.33$ in [1,3] and its independence with regard to large scales' initial conditions $\sigma$ : in particular, the latter point means that at sufficiently large St or Reynolds numbers, the growth rate is similar for Saffman $(\sigma=2)$ and Batchelor $(\sigma=4)$ turbulence.

In a sustained shear flow, kinetic energy is continuously produced by the mean-velocity gradient and verifies the evolution equation

$$
\frac{\mathrm{d} K}{\mathrm{~d} t}=S R_{13}(t)-\epsilon(t), \quad \frac{\mathrm{d} U_{1}}{\mathrm{~d} x_{3}}=-S, \quad A_{i j}=\frac{\mathrm{d} U_{i}}{\mathrm{~d} x_{j}},
$$

where $\epsilon$ is the kinetic energy dissipation rate. Kinetic energy eventually grows exponentially $[27,31,32]$ at a rate $\gamma=2 b_{13}-\epsilon / K S$ when the global anisotropy indicators $b_{i j}$ have reached an asymptotic state.

Values of $\gamma$ obtained in experiments [33-35] and in DNS [10,11,17,20,36,37] are smaller than the $\gamma=0.33$ obtained with the present anisotropic EDQNM modelling, and some values are reported in Table 1 of [3]. In what follows, we first bring theoretical arguments which were not provided in $[1,3]$ to justify the value $\gamma=0.33$ obtained with the model. To this end, we use the methodology of Soulard et al. [38] who derived a prediction for the growth rate of the Rayleigh-Taylor mixing zone length as a function of the infrared slope $\sigma$. These theoretical arguments were also applied to the framework of unstably stratified homogeneous turbulence (USHT) in $[4,5,38]$ to determine the exponential growth rate of the kinetic energy: results will be discussed hereafter.

The demonstration essentially relies on two features: (i) the linear dynamics of large scales dominated by the anisotropy production and (ii) the self-similarity of the kinetic energy spectrum. In the self-similar state, the kinetic energy and its dissipation rate evolve as $K \sim \epsilon \sim \exp (\gamma S t)$ : combining this with dimensional analysis for the integral scale $L \sim$ $K^{3 / 2} / \epsilon$ yields $L \sim \exp (\gamma S t / 2)$. Let us call $\gamma_{E}$ the exponential growth rate of the kinetic energy spectrum large scales. Assuming that the main contribution to kinetic energy comes from large scales, one has

$$
K(t)=\int_{0}^{\infty} E(k, t) \mathrm{d} k \simeq \int_{0}^{k_{L}} k^{\sigma} \exp \left(\gamma_{E} S t\right) \mathrm{d} k=\frac{k_{L}^{\sigma+1}}{\sigma+1} \exp \left(\gamma_{E} S t\right) .
$$

Further using the self-similarity hypothesis for the kinetic energy and the integral scale $k_{L} \sim 1 / L \sim \exp (-\gamma S t / 2)$, one obtains

$$
\gamma_{E}=\frac{\gamma}{2}(\sigma+3)
$$

This result can also be obtained differently, in a manner analogous to [32]: assuming that at large scales the kinetic energy is given by $K \sim S^{2} L^{2}$, one can expand the kinetic energy spectrum according to

$$
E\left(k<k_{L}, t\right)=K(t) L(t)(k L(t))^{\sigma}=S^{2} L(t)^{\sigma+3} k^{\sigma} \sim S^{2} \exp \left[\frac{\sigma+3}{2} \gamma S t\right] k^{\sigma},
$$


which further illustrates that the self-similar evolution of the integral scale is crucial in the demonstration. It is worth noting that these two equations hold for both shear flows and USHT, if one replaces $S$ by the stratification frequency in the latter case.

Then, the exponential growth rate $\gamma_{E}$ is also given by the largest eigenvalue $\Gamma_{\max }$ of the linear operator of the generalised Lin equations system (18), (19), and (20). The linear operator of shear flows verifies, dropping the non-linear and viscous terms and using the expression (25) of self-similarity

$$
\begin{aligned}
& \frac{\partial}{\partial t}\left(\begin{array}{c}
E \\
E H_{11}^{(\mathrm{dir})} \\
E H_{13}^{(\mathrm{dir})} \\
E H_{33}^{(\mathrm{dir})} \\
E H_{11}^{(\mathrm{pol})} \\
E H_{13}^{(\mathrm{pol})} \\
E H_{33}^{(\mathrm{pol})}
\end{array}\right) \\
& =S\left(\begin{array}{ccccccc}
0 & 0 & 2(\sigma+2) & 0 & 0 & 2 & 0 \\
0 & 0 & -\frac{2(\sigma+11)}{21} & 0 & 0 & \frac{2}{21} & 0 \\
\frac{(\sigma-1)}{30} & \frac{3-\sigma}{7} & 0 & -\frac{4+\sigma}{7} & \frac{1}{7} & 0 & \frac{1}{7} \\
0 & 0 & \frac{2(10-\sigma)}{21} & 0 & 0 & \frac{2}{21} & 0 \\
0 & 0 & \frac{4}{7} & 0 & 0 & \frac{2(\sigma+4)}{21} & 0 \\
\frac{1}{5} & \frac{6}{7} & 0 & \frac{6}{7} & \frac{3 \sigma-2}{21} & 0 & \frac{3 \sigma+5}{21} \\
0 & 0 & \frac{4}{7} & 0 & 0 & \frac{2(\sigma-3)}{21} & 0
\end{array}\right)\left(\begin{array}{c}
E \\
E H_{11}^{(\mathrm{dir})} \\
E H_{13}^{(\mathrm{dir})} \\
E H_{33}^{(\mathrm{dir})} \\
E H_{11}^{(\mathrm{pol})} \\
E H_{13}^{(\mathrm{pol})} \\
E H_{33}^{(\mathrm{pol})}
\end{array}\right) .
\end{aligned}
$$

Note that (i) the spanwise component does not appear since $H_{22}=-H_{11}-H_{33}$ because of incompressibility and (ii) each component of $E H_{i j}$ was assumed to behave spatially at large scales like $\sim k^{\sigma}$, which is verified numerically. The maximum real eigenvalue $\Gamma_{\max }(\sigma)$ of the linear operator is given by

$$
\begin{aligned}
\Gamma_{\max }(\sigma)= & \frac{1}{21 \sqrt{10}}[-1112+267 \sigma(1+\sigma) \\
& \left.+49 \sqrt{3664-168 \sigma-159 \sigma^{2}+18 \sigma^{3}+9 \sigma^{4}}\right]^{1 / 2} .
\end{aligned}
$$

The variations of $\Gamma_{\max }$ as a function of $\sigma$ are presented in Figure 1(a) (dashed line): one can note that for common values of the infrared slope, basically from $\sigma=1$ to $\sigma=4$, there is a strong variation of $\Gamma_{\max }$. Recall that $\Gamma_{\max }$ was obtained by assuming that the dynamics of the large scales is linear: this is further assessed in Figure 1(b) where the budget terms of the evolution equation (18) of $E(k, t)$ are displayed: it is clear that at large scales the linear production term is much larger than the non-linear transfer term. Figure 1(b) further shows two features: (i) at small scales, before the Kolmogorov wavenumber $k_{\eta}$, nonlinear transfers balance dissipation. And (ii) the linear mechanisms are the most intense for $k_{L}<k<k_{S}$, where $k_{S}=\sqrt{S^{3} / \epsilon}$ is the shear wavenumber. The dominance of linear terms at large scales can also be observed in Figure 1(c), where the absolute values of the 


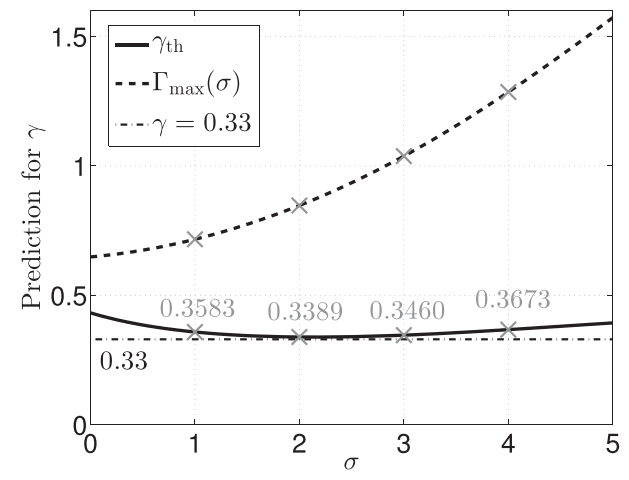

(a)

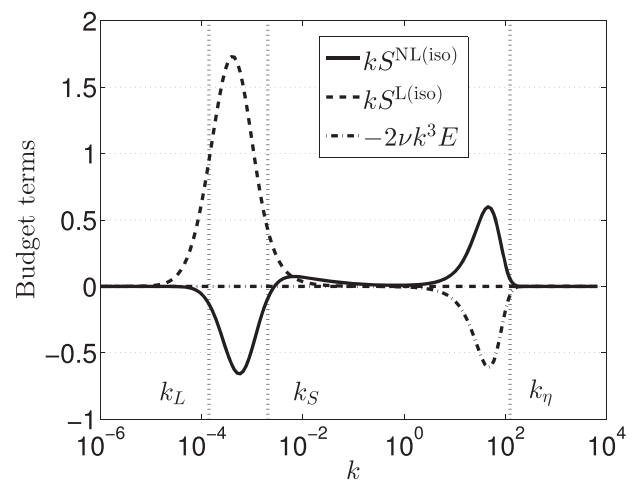

(b)

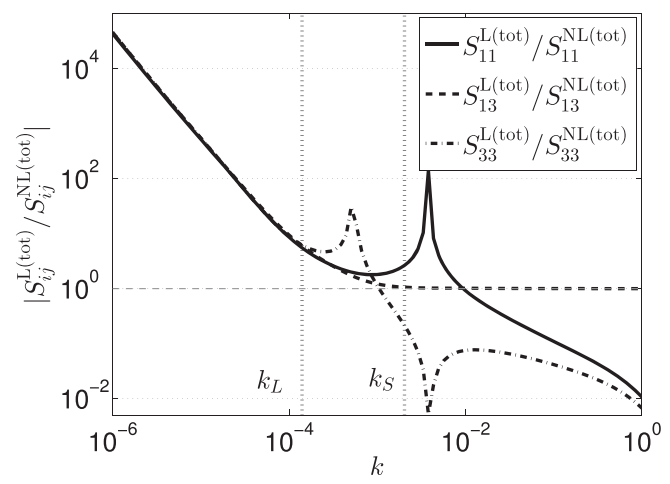

(c)

Figure 1. (a) Theoretical prediction for the kinetic energy exponential growth rate $\gamma_{\text {th }}=$ $2 \Gamma_{\max }(\sigma) /(\sigma+3)$, where $\Gamma_{\max }$ is provided in Equation (27). The numerical values in grey represent the expected $\gamma_{\text {th }}$ for the classical integer values of the infrared slope $\sigma$, whereas the dash-dot line represents the value obtained by the anisotropic EDQNM modelling $\gamma=0.33$. (b) Budget terms of the equation of $E(k, t)$ given in Equation (18), for $\sigma=2$ and $\operatorname{Re}_{\lambda}(S t=50)=2.4 \times 10^{4}$, normalised by $\epsilon$, along with the integral, shear and Kolmogorov wavenumbers $k_{L}, k_{S}$, and $k_{\eta}$. (c) Ratio of the linear to non-linear transfers, for the same configuration as (b). The total transfer is given by $S_{i j}^{(\text {tot })}=2\left(\delta_{i j}{ }^{(\text {iso })} / 3+S_{i j}^{(\text {dir })}+S_{i j}^{(\text {pol })}\right)$.

linear to non-linear terms ratios are presented for the components ()$_{11},()_{13}$, and ()$_{33}$. On the contrary, for $k>k_{S}$, the non-linear transfers drive the dynamics, as observed by values of the ratios much lower than unity.

The final theoretical prediction for the kinetic energy exponential growth rate $\gamma_{\text {th }}$ is obtained by equating the most amplified eigenvalue and the kinetic energy spectrum growth rate

$$
\gamma_{E}=\Gamma_{\max }(\sigma) \Leftrightarrow \gamma_{\text {th }}=\frac{2 \Gamma_{\max }(\sigma)}{\sigma+3},
$$

and is presented in Figure 1(a), where it is clear that the true exponential growth rate of the kinetic energy ( - curve) accounting for non-linearities is much lower than the linear prediction (-- curve), and almost independent of the large scales initial conditions $\sigma$. In 
particular, one has for the integer values of the infrared slope $\gamma_{\text {th }}(\sigma=1)=0.358, \gamma_{\text {th }}(\sigma=$ $2)=0.339, \gamma_{\text {th }}(\sigma=3)=0.346$, and $\gamma_{\text {th }}(\sigma=4)=0.367$.

The implications of Equation (28) and the numerical results in Figure 1(a) are twofold. First, there is a good agreement between the new theoretical prediction (28) - which relies on the linear dynamics at large scales and the self-similarity of the kinetic energy spectrum - and the numerical simulations of [1,3], which gave a value around $0.33 \leq \gamma \leq 0.34$, as recalled for clarity in Figure 3(a) in grey.

Secondly, one can remark that the predictions for the exponential growth rate hardly depend on $\sigma$ in Figure 1(a), which is also in agreement with the simulations of [3]. This is of great theoretical interest because it strongly differs from USHT, where kinetic energy also grows exponentially, but with a strong dependence on large scales according to the theoretical prediction [5]

$$
\gamma_{\mathrm{USHT}}=\frac{4}{\sigma+3}
$$

where the kinetic energy exponential growth rate $\gamma$ USHT varies from 1 to $4 / 7$ for $\sigma=1$ up to $\sigma=4$. It is worth noting that the present model is able to capture the strong dependence of the kinetic energy exponential growth rate in USHT as well [4]. The reason why the asymptotic anisotropic states strongly differ between shear flows and USHT is because the linear operator of the production terms does not depend on $\sigma$ in the latter case. Indeed, the $\sigma$-contributions in the linear operator of shear flows appear due to the spatial-derivative terms in $\partial / \partial k$ (see Appendix 1), which arise from the mean-flow advection term in the Craya equation (2): basically, they balance the $\sigma$-dependence of $\gamma_{E}$.

In conclusion, it has been shown analytically within the anisotropic EDQNM modelling that the kinetic energy exponential growth rate in shear flows is independent of the large scales initial conditions $\sigma$, consistently with self-similarity arguments. This independence strongly differs from the asymptotic exponential growth rate in USHT which depends on $\sigma$. The model is able to recover both phenomenologies: this is the first theoretical contribution of this paper.

\subsection{Quadratic contributions of anisotropy}

For the modelling of non-linear transfers, it was assumed for simplicity reasons in $[1,3]$ that quadratic contributions of anisotropy could be discarded, namely contributions like $H_{i l}^{(\mathrm{dir})} H_{l j}^{(\mathrm{dir})}, H_{i l}^{(\mathrm{dir})} H_{l j}^{(\mathrm{pol})}, \ldots$ and so on. In this part, we verify this assumption. The four corresponding quadratic non-linear transfers were explicitly computed and are gathered in Appendix 2 because the expressions are rather lengthy: the isotropic one $Q^{\mathrm{NL}(\mathrm{iso})}$, the directional and polarisation ones $Q_{i j}^{\mathrm{NL}(\mathrm{dir})}$ and $Q_{i j}^{\mathrm{NL}(\mathrm{pol})}$, and the return-to-isotropy one $Q_{i j}^{\mathrm{NL}(\mathrm{RTI})}$, which should be respectively added to $S^{\mathrm{NL}(\mathrm{iso})}, S_{i j}^{\mathrm{NL}(\mathrm{dir})}, S_{i j}^{\mathrm{NL}(\mathrm{pol})}$, and $S_{i j}^{(\mathrm{RTI})}$, the latter being defined in Appendix 1. The isotropic term $Q^{\mathrm{NL}(\text { iso) }}$ is conservative, and one has

$$
\int_{0}^{\infty}\left[2\left(Q^{\mathrm{NL}(\mathrm{iso})} \frac{\delta_{i j}}{3}+Q_{i j}^{\mathrm{NL}(\mathrm{dir})}+Q_{i j}^{\mathrm{NL}(\mathrm{pol})}\right)-Q_{i j}^{\mathrm{NL}(\mathrm{RTI})}\right] \mathrm{d} k=0 .
$$

In fact, we derived analytically $Q_{i j}^{\mathrm{NL}(\mathrm{RTI})}$ only to check the latter property, which is a good way to identify calculations errors. The impact of quadratic anisotropic contributions on 


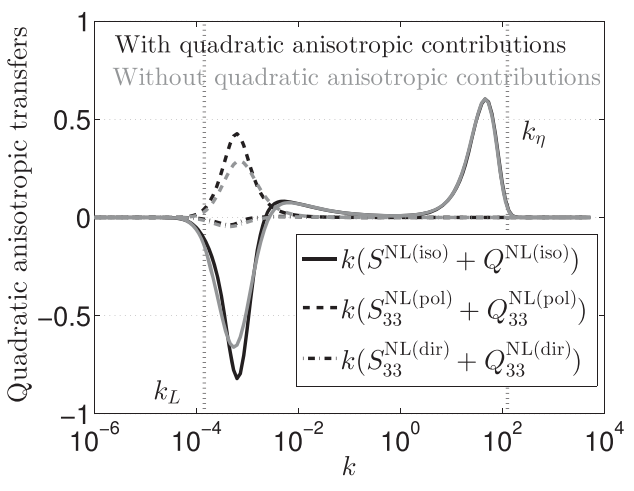

(a)

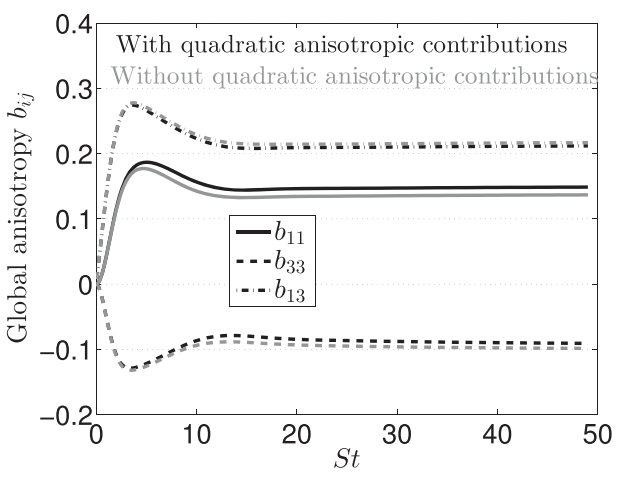

(b)

Figure 2. Quadratic anisotropic contributions in the non-linear transfers at $S t=50$, with $\sigma=2$. Grey curves represent simulations without the quadratic anisotropic contributions. (a) Budget terms along with the integral and Kolmogorov wavenumbers $k_{L}$ and $k_{\eta}$. (b) Global anisotropy indicator $b_{i j}$.

the non-linear transfers is revealed in Figure 2(a). One can note that the global shape of the transfers is preserved and that there is a rather small change in intensity, mainly at large scales which is expected since this is where anisotropy is dominant. The isotropic and polarisation parts of the transverse component ()$_{33}$ are more affected by the quadratic contributions than the directional part.

In a recent work by Soulard and Gréa [39], a zero-mode analysis was performed on a distinct anisotropic EDQNM model restricted to axisymmetric configurations. It was notably found that zero modes of higher-order harmonics, which could be compared to the quadratic anisotropic contributions here, for example, become negligible for increasing $k$. This is fully consistent with what is obtained in Figure 2(a), namely that the quadratic anisotropic contributions are completely negligible at small scales.

Regarding the global anisotropy indicator $b_{i j}$ in Figure 2(b): the asymptotic values are almost the same with and without the quadratic anisotropic contributions. One can observe a slight increase of the streamwise anisotropy and decrease of the transverse one. The kinetic energy exponential growth rate is not modified by the presence of the quadratic anisotropic contributions.

In conclusion, the quite heavy analytical calculations which led to the quadratic anisotropic contributions in the non-linear transfers do not provide significant changes and lengthen the numerical simulations. This fully justifies that they were neglected so far. This analysis also indicates that in order to improve the modelling of anisotropy, it is not efficient to refine the non-linear transfers, which remains a noticeable feature: one should rather improve the production terms, which is the topic of the next part.

\section{Modelling with the fourth-order expansion of $\mathcal{E}$ and $Z$}

In this section, we aim at improving the anisotropic EDQNM modelling originally developed in MCS by considering the fourth-order terms in the expansions for $\mathcal{E}$ and $Z$ in Equations (9) and (10), respectively. The objective is rather simple, namely to show that taking into account more spherical harmonics improves the results of the model for shear 
flows, and more precisely decreases the value of the kinetic energy exponential growth rate $\gamma$. To this end, the theoretical developments necessary to the fourth-order expansion are exposed, and then the new spherically averaged equations are derived.

\subsection{The operators of the fourth-order expansion}

The first step is to determine the two fourth-order operators $P_{i j p q}$ and $N_{i j p q}$ which, when contracted with $\mathcal{E}$ and $Z$ respectively, extract only the fourth-order tensors $U_{i j p q}^{(\mathrm{dir}) 4}$ and $U_{i j p q}^{(\mathrm{pol}) 4}$ of Equations (9) and (10), and erase the $H_{i j}^{(\mathrm{dir})}$ and $H_{i j}^{(\mathrm{pol})}$ contributions. This is similar to what was done for the second-order expansion in [12], where the operators are, according to Equations (11) and (12)

$$
2 E H_{i j}^{(\mathrm{dir})}=\int_{S_{k}} \mathcal{E}\left(\frac{\delta_{i j}}{3}-\alpha_{i} \alpha_{j}\right) \mathrm{d}^{2} \boldsymbol{k}, \quad 2 E H_{i j}^{(\mathrm{pol})}=\int_{S_{k}} \Re\left(Z N_{i} N_{j}\right) \mathrm{d}^{2} \boldsymbol{k} .
$$

The operator $P_{i j p q}$ is given in the recent study of Rubinstein et al. [23]. On the other hand, $N_{i j p q}$ was not and is more complex to handle since it involves both the normalised wavevector $\alpha=k / k$ and the helical mode $N$. After some algebra, one gets

$$
\begin{gathered}
P_{i j p q}=\alpha_{i} \alpha_{j} \alpha_{p} \alpha_{q}-\frac{1}{7}\left(\delta_{i j} \alpha_{p} \alpha_{q}+5 \text { perm. }\right)+\frac{1}{35}\left(\delta_{i j} \delta_{p q}+\delta_{i p} \delta_{j q}+\delta_{i q} \delta_{j p}\right), \\
N_{i j p q}=\left(N_{i} N_{j} \alpha_{p} \alpha_{q}+N_{p} N_{q} \alpha_{i} \alpha_{j}+4 \text { perm. }\right)-\frac{1}{7}\left(\delta_{i j} N_{p} N_{q}+5 \text { perm. }\right),
\end{gathered}
$$

where 'perm.' refers to the other permutations of indices $i, j$, $p$, and $q$ not written explicitly for the sake of brevity. Thanks to these two operators, one defines the fourth-order spectral anisotropy descriptors $H_{i j p q}^{(\mathrm{dir})}$ and $H_{i j p q}^{(\mathrm{pol})}$, similarly to $H_{i j}^{(\mathrm{dir})}$ and $H_{i j}^{(\mathrm{pol})}$ in Equation (31), as

$$
\begin{gathered}
2 E(k, t) H_{i j p q}^{(\mathrm{dir})}(k, t)=\int_{S_{k}} \mathcal{E}(\boldsymbol{k}, t) P_{i j p q}(\boldsymbol{k}) \mathrm{d}^{2} \boldsymbol{k}=\frac{24}{945} E(k, t) U_{i j p q}^{(\mathrm{dir}) 4}(k, t), \\
2 E(k, t) H_{i j p q}^{(\mathrm{pol})}(k, t)=\int_{S_{k}} \Re\left(Z(\boldsymbol{k}, t) N_{i j p q}(\boldsymbol{k})\right) \mathrm{d}^{2} \boldsymbol{k}=\frac{4}{21} E(k, t) U_{i j p q}^{(\mathrm{pol}) 4}(k, t),
\end{gathered}
$$

so that the fourth-order expansion of $\mathcal{E}$ and $Z$ reads

$$
\begin{gathered}
\mathcal{E}(\boldsymbol{k}, t)=\mathcal{E}_{0}\left(1-15 H_{i j}^{(\mathrm{dir})}(k, t) \alpha_{i} \alpha_{j}+\frac{945}{12} H_{i j p q}^{(\mathrm{dir})}(k, t) \alpha_{i} \alpha_{j} \alpha_{p} \alpha_{q}\right), \\
Z(\boldsymbol{k}, t)=\frac{1}{2} \mathcal{E}_{0}\left(5 H_{i j}^{(\mathrm{pol})}(k, t)+\frac{21}{2} H_{i j p q}^{(\mathrm{pol})}(k, t) \alpha_{p} \alpha_{q}\right) N_{i}^{*} N_{j}^{*} .
\end{gathered}
$$

At this point, some words need to be said about the properties of the new fourth-order tensors and related assumptions.

One can remark that at the fourth order, the polarisation part $H_{i j p q}^{(\mathrm{pol})}$ is contracted with both normalised wavevectors $\alpha_{p} \alpha_{q}$ and helical modes $N_{i}^{*} N_{j}^{*}$, unlike the directional part 
$H_{i j p q}^{(\mathrm{dir})}$ which is only contracted with $\alpha_{i} \alpha_{j} \alpha_{p} \alpha_{q}$. Hence, $H_{i j p q}^{(\mathrm{dir})}$ is fully symmetric in its indices, as reported in [23]. The latter property of full symmetry is thus a priori not verified by $H_{i j p q}^{(\mathrm{pol})}$, but we nonetheless made this assumption. Otherwise, multiple independent variables related to the fourth-order polarisation appear in the calculations which cannot be dealt with in this framework. In addition, we assumed that $H_{i j p q}^{(\mathrm{pol})}$ is trace-free, meaning that any contraction of two indices yields zero. For $H_{i j p q}^{(\mathrm{dir})}$, this is verified according to [23], but for $H_{i j p q}^{(\mathrm{pol})}$ this is a supplementary assumption. These two assumptions for $H_{i j p q}^{(\mathrm{pol})}$ were used to obtain Equation (35) and thus Equation (37).

Consequently, there is room for improvement for the modelling of the fourth-order expansion of $Z$. Nevertheless, the present assumptions for $H_{i j p q}^{(\mathrm{pol})}$ allow to close the different equations, and the numerical results presented in Section 3.5 show that these hypotheses are acceptable. It is not clear what different rules could be used for $H_{i j p q}^{(\mathrm{pol})}$ and this is an open question: one could think of a restriction with distinct $i \leftrightarrow j$ and $p \leftrightarrow q$ symmetries; the contractions $i=p, i=q, j=p, j=q$ could also be discussed.

With the fourth-order expansion, the modelled expression (15) of the spectral Reynolds tensor $\hat{R}_{i j}$ now reads

$$
\begin{aligned}
\hat{R}_{i j}= & \underbrace{\mathcal{E}_{0} P_{i j}\left(1-15 H_{p q}^{(\mathrm{dir})} \alpha_{p} \alpha_{q}+\frac{945}{12} H_{r s p q}^{(\mathrm{dir})} \alpha_{r} \alpha_{s} \alpha_{p} \alpha_{q}\right)}_{\hat{R}_{i j}^{\mathrm{e} 2}+\hat{R}_{i j}^{\mathrm{e} 4}} \\
& +\underbrace{\frac{1}{2} \mathcal{E}_{0}\left(5 H_{p q}^{(\mathrm{pol})}+\frac{21}{2} H_{p q r s}^{(\mathrm{pol})} \alpha_{r} \alpha_{s}\right)\left(2 P_{i p} P_{j q}+P_{i j} \alpha_{p} \alpha_{q}\right)}_{\hat{R}_{i j}^{\mathrm{z}}+\hat{R}_{i j}^{\mathrm{z}}} .
\end{aligned}
$$

It is worth noting that the spherical average of $\hat{R}_{i j}$ remains unchanged whether the fourthorder contributions are considered or not, i.e. Equation (13) is still true. The final step of the fourth-order modelling is to determine analytically (i) the linear and non-linear transfers associated with $E H_{i j p q}^{(\mathrm{dir})}$ and $E H_{i j p q}^{(\mathrm{pol})}$, and (ii) the retro-action of the fourth-order contributions on the equations of $E, E H_{i j}^{(\mathrm{dir})}$, and $E H_{i j}^{(\mathrm{pol})}$. For the sake of readability, no intermediate steps are given here since the expressions are very lengthy, but they can be found in [40].

\subsection{Fourth-order productions terms}

In this part, we aim at determining the explicit expressions of the production terms, linear with the mean-velocity gradient matrix $A_{i j}$, linked to the fourth-order contributions in the expansions (36) and (37) of $\mathcal{E}$ and $Z$, respectively. They are of three kinds: the fourthorder contributions in the equations of $E H_{i j}^{(\mathrm{dir})}$ and $E H_{i j}^{(\mathrm{pol})}$, in the equations of $E H_{i j p q}^{(\mathrm{dir})}$ and $E H_{i j p q}^{(\mathrm{pol})}$, and the second-order contributions in the equations of $E H_{i j p q}^{(\mathrm{dir})}$ and $E H_{i j p q}^{(\mathrm{pol})}$. From the properties of $H_{i j p q}^{(\mathrm{dir})}$ and $H_{i j p q}^{(\mathrm{pol})}$, it follows that there are no fourth-order contributions in the equation for $E$. 
First, the contributions of the fourth-order expansion in the equations of $E H_{i j}^{(\mathrm{dir})}$ and $E H_{i j}^{(\mathrm{pol})}$ are, after some algebra,

$$
\begin{gathered}
S_{i j}^{\mathrm{L}(\mathrm{dir} 4)}(k, t)=-3 A_{l n}^{+} E H_{i j l n}^{(\mathrm{dir})}-A_{l n}^{+} \frac{\partial\left(k E H_{i j l n}^{(\mathrm{dir})}\right)}{\partial k}+\frac{1}{6} A_{l n}^{+} E H_{i j l n}^{(\mathrm{pol})}, \\
S_{i j}^{\mathrm{L}(\mathrm{pol} 4)}(k, t)=-A_{l n}^{+} E H_{i j l n}^{(\mathrm{dir})}+\frac{1}{2} A_{l n}^{+} E H_{i j l n}^{(\mathrm{pol})}+\frac{1}{6} A_{l n}^{+} \frac{\partial\left(k E H_{i j l n}^{(\mathrm{pol})}\right)}{\partial k},
\end{gathered}
$$

where the superscripts (dir4) and (pol4) refer to the contributions of the fourth-order expansion. Consequently and for the sake of clarity, the linear transfers of Equations (19) and (20) are renamed $S_{i j}^{\mathrm{L}(\mathrm{dir} 2)}$ and $S_{i j}^{\mathrm{L}(\text { pol2) }}$, so that the total linear transfers for $E H_{i j}^{(\mathrm{dir})}$ and $E H_{i j}^{(\mathrm{pol})}$ are $S_{i j}^{\mathrm{L}(\mathrm{dir})}=S_{i j}^{\mathrm{L}(\mathrm{dir} 2)}+S_{i j}^{\mathrm{L}(\mathrm{dir} 4)}$ and similarly for $S_{i j}^{\mathrm{L}(\mathrm{pol})}$.

Now, we define the linear directional and polarisation production terms $S_{i j p q}^{\mathrm{L}(\mathrm{dir})}$ and $S_{i j p q}^{\mathrm{L}(\mathrm{pol})}$ of $E H_{i j p q}^{(\mathrm{dir})}$ and $E H_{i j p q}^{(\mathrm{pol})}$, which can also be divided into two terms, resulting respectively from the second- and fourth-order contributions

$$
\begin{gathered}
S_{i j p q}^{\mathrm{L}(\mathrm{dir})}=\frac{1}{4} \int_{S_{k}}\left(A_{l n} k_{l} \frac{\partial \hat{R}_{m m}}{\partial k_{n}}-2 M_{m n} \hat{R}_{n m}\right) P_{i j p q} \mathrm{~d}^{2} \boldsymbol{k}, \\
S_{i j p q}^{\mathrm{L}(\mathrm{pol})}=\frac{1}{4} \int_{S_{k}} \Re\left[\left(A_{\ln } k_{l} \frac{\partial \hat{R}_{r s}}{\partial k_{n}}-M_{r n} \hat{R}_{n s}-M_{s n} \hat{R}_{n r}\right) N_{r}^{*} N_{s}^{*} N_{i j p q}\right] \mathrm{d}^{2} \boldsymbol{k} .
\end{gathered}
$$

First, we determine the impact of the second-order contributions $E H_{i j}^{(\mathrm{dir})}$ and $E H_{i j}^{(\mathrm{pol})}$ on the linear terms of $E H_{i j p q}^{(\mathrm{dir})}$ and $E H_{i j p q}^{(\mathrm{pol})}$. For this purpose, it is convenient to introduce two symmetric and trace-free operators $\mathcal{H}_{i j p q}^{(2, \mathrm{e})}\left[E H^{()}\right]$and $\mathcal{H}_{i j p q}^{(2, z)}\left[E H^{()}\right]$, which refer to terms involving respectively $\hat{R}_{i j}^{\mathrm{e} 2}$ and $\hat{R}_{i j}^{\mathrm{z} 2}$ of Equation (15), namely

$$
\begin{aligned}
\mathcal{H}_{i j p q}^{(2, \mathrm{e})}\left[E H^{()}\right]= & E\left[\frac{8}{5} A_{l n}^{+} H_{l n}^{()}\left(\delta_{i j} \delta_{p q}+\delta_{i p} \delta_{j q}+\delta_{i q} \delta_{j p}\right)-4\left[\delta_{i j}\left(A_{l p}^{+} H_{l q}^{()}+A_{l q}^{+} H_{l p}^{()}\right)\right.\right. \\
& +\delta_{p q}\left(A_{l i}^{+} H_{l j}^{()}+A_{l j}^{+} H_{l i}^{()}\right)+A_{l p}^{+}\left(H_{l j}^{()} \delta_{i q}+H_{l i}^{()} \delta_{j q}\right)+A_{l q}^{+}\left(H_{l j}^{()} \delta_{i p}+H_{l i}^{()} \delta_{j p}\right) \\
& \left.+H_{l p}^{(O}\left(A_{j l}^{+} \delta_{i q}+A_{i l}^{+} \delta_{j q}\right)+H_{l q}^{()}\left(A_{j l}^{+} \delta_{i p}+A_{i l}^{+} \delta_{j p}\right)\right] \\
& \left.+14\left(A_{i j}^{+} H_{p q}^{()}+A_{p q}^{+} H_{i j}^{()}+A_{i p}^{+} H_{j q}^{()}+A_{i q}^{+} H_{j p}^{()}+A_{j p}^{+} H_{i q}^{()}+A_{j q}^{+} H_{i p}^{()}\right)\right], \quad(43) \\
\mathcal{H}_{i j p q}^{(2, \mathrm{z})}\left[E H^{()}\right]= & E\left[4 A_{l n}^{+} H_{l n}^{()}\left(\delta_{i j} \delta_{p q}+\delta_{i p} \delta_{j q}+\delta_{i q} \delta_{j p}\right)-10\left[\delta_{i j}\left(A_{l p}^{+} H_{l q}^{()}+A_{l q}^{+} H_{l p}^{()}\right)\right.\right. \\
& +\delta_{p q}\left(A_{l i}^{+} H_{l j}^{()}+A_{l j}^{+} H_{l i}^{()}\right)+A_{l p}^{+}\left(H_{l j}^{()} \delta_{i q}+H_{l i}^{()} \delta_{j q}\right)+A_{l q}^{+}\left(H_{l j}^{()} \delta_{i p}+H_{l i}^{()} \delta_{j p}\right) \\
& \left.+H_{l p}^{()}\left(A_{j l}^{+} \delta_{i q}+A_{i l}^{+} \delta_{j q}\right)+H_{l q}^{()}\left(A_{j l}^{+} \delta_{i p}+A_{i l}^{+} \delta_{j p}\right)\right] \\
& \left.+35\left(A_{i j}^{+} H_{p q}^{()}+A_{p q}^{+} H_{i j}^{()}+A_{i p}^{+} H_{j q}^{()}+A_{i q}^{+} H_{j p}^{()}+A_{j p}^{+} H_{i q}^{()}+A_{j q}^{+} H_{i p}^{()}\right)\right] . \quad(44)
\end{aligned}
$$


After some algebra, one eventually obtains

$$
\begin{gathered}
S_{i j p q}^{\mathrm{L}(\mathrm{dir} 2)}=\frac{1}{441}\left(-\frac{1}{6} \mathcal{H}_{i j p q}^{(2, \mathrm{e})}\left[E H^{(\mathrm{pol})}\right]+4 \mathcal{H}_{i j p q}^{(2, \mathrm{e})}\left[E H^{(\mathrm{dir})}\right]-\mathcal{H}_{i j p q}^{(2, \mathrm{e})}\left[\partial_{k}\left(k E H^{(\mathrm{dir})}\right)\right]\right), \\
S_{i j p q}^{\mathrm{L}(\mathrm{pol} 2)}=\frac{1}{441}\left(6 \mathcal{H}_{i j p q}^{(2, \mathrm{z})}\left[E H^{(\mathrm{dir})}\right]-4 \mathcal{H}_{i j p q}^{(2, \mathrm{z})}\left[E H^{(\mathrm{pol})}\right]+\mathcal{H}_{i j p q}^{(2, \mathrm{z})}\left[\partial_{k}\left(k E H^{(\mathrm{pol})}\right)\right]\right) .
\end{gathered}
$$

Consistently with the previous calculations, the superscripts (dir2) and (pol2) refer to the contributions of the second-order expansion. Finally, we proceed similarly to determine the impact of the fourth-order contributions on the linear terms of $E H_{i j p q}^{(\mathrm{dir})}$ and $E H_{i j p q}^{(\mathrm{pol})}$. It is also convenient to define a final operator $\mathcal{H}_{i j p q}^{(4)}\left[E H^{()}\right]$which reflects the terms coming from $\hat{R}_{i j}^{\mathrm{e} 4}$ and $\hat{R}_{i j}^{\mathrm{z} 4}$ of Equation (38):

$$
\begin{aligned}
\mathcal{H}_{i j p q}^{(4)}\left[E H^{()}\right]= & E\left[A_{l i}^{+} H_{j l p q}^{()}+A_{l j}^{+} H_{i l p q}^{()}+A_{l p}^{+} H_{i j l q}^{()}+A_{l q}^{+} H_{i j l p}^{()}\right. \\
& \left.-\frac{2}{7} A_{l n}^{+}\left(\delta_{i j} H_{l n p q}^{()}+\delta_{p q} H_{l n i j}^{()}+\delta_{i p} H_{l n j q}^{()}+\delta_{i q} H_{l n j p}^{()}+\delta_{j p} H_{l n i q}^{()}+\delta_{j q} H_{l n i p}^{()}\right)\right] .
\end{aligned}
$$

After intricate algebra involving the spherical integration of terms containing 10 normalised wavevectors $\alpha$, one finally gets

$$
\begin{aligned}
S_{i j p q}^{\mathrm{L}(\mathrm{dir} 4)}= & \frac{1}{11}\left(2 \mathcal{H}_{i j p q}^{(4)}\left[\partial_{k}\left(k E H^{(\mathrm{dir})}\right)\right]-\mathcal{H}_{i j p q}^{(4)}\left[E H^{(\mathrm{dir})}\right]+\frac{3}{5} \mathcal{H}_{i j p q}^{(4)}\left[E H^{(\mathrm{pol})}\right]\right) \\
& +E\left(A_{i l}^{-} H_{j p q l}^{(\mathrm{dir})}+A_{j l}^{-} H_{i p q l}^{(\mathrm{dir})}+A_{p l}^{-} H_{i j l q}^{(\mathrm{dir})}+A_{q l}^{-} H_{i j l p}^{(\mathrm{dir})}\right), \\
S_{i j p q}^{\mathrm{L}(\mathrm{pol} 4)}= & \frac{1}{11}\left(\frac{4}{5} \mathcal{H}_{i j p q}^{(4)}\left[\partial_{k}\left(k E H^{(\mathrm{pol})}\right)\right]-\frac{2}{5} \mathcal{H}_{i j p q}^{(4)}\left[E H^{(\mathrm{pol})}\right]+54 \mathcal{H}_{i j p q}^{(4)}\left[E H^{(\mathrm{dir})}\right]\right) \\
& +\frac{3}{5} E\left(A_{i l}^{-} H_{j p q l}^{(\mathrm{pol})}+A_{j l}^{-} H_{i p q l}^{(\mathrm{pol})}+A_{p l}^{-} H_{i j l q}^{(\mathrm{pol})}+A_{q l}^{-} H_{i j l p}^{(\mathrm{pol})}\right) .
\end{aligned}
$$

The total fourth-order directional and polarisation linear production terms are consequently $S_{i j p q}^{\mathrm{L}(\mathrm{dir})}=S_{i j p q}^{\mathrm{L}(\mathrm{dir} 2)}+S_{i j p q}^{\mathrm{L}(\mathrm{dir} 4)}$, and similarly for $S_{i j p q}^{\mathrm{L}(\mathrm{pol})}$.

\subsection{Fourth-order non-linear transfer terms}

The fourth-order non-linear directional and polarisation transfers $S_{i j p q}^{\mathrm{NL}(\mathrm{dir})}$ and $S_{i j p q}^{\mathrm{NL}(\mathrm{pol})}$ are defined as

$$
\begin{gathered}
S_{i j p q}^{\mathrm{NL}(\mathrm{dir})}(k, t)=\frac{1}{2} \int_{S_{k}} T_{\mathcal{E}}(\boldsymbol{k}, t) P_{i j p q}(\boldsymbol{k}) \mathrm{d}^{2} \boldsymbol{k}, \\
S_{i j p q}^{\mathrm{NL}(\mathrm{pol})}(k, t)=\frac{1}{2} \int_{S_{k}} \Re\left(T_{Z}(\boldsymbol{k}, t) N_{i j p q}(\boldsymbol{k})\right) \mathrm{d}^{2} \boldsymbol{k} .
\end{gathered}
$$

Discarding the quadratic contributions of anisotropy as for the second-order expansions, one obtains the important fact that $S_{i j p q}^{\mathrm{NL}(\mathrm{dir})}$ and $S_{i j p q}^{\mathrm{NL}(\mathrm{pol})}$ only depend on the fourth-order 
contributions. In addition, since $H_{i j p q}^{(\mathrm{dir})}$ and $H_{i j p q}^{(\mathrm{pol})}$ are symmetric and trace-free, it follows that fourth-order contributions vanish in $S_{i j}^{\mathrm{NL}(\mathrm{dir})}$ and $S_{i j}^{\mathrm{NL}(\mathrm{pol})}$. The calculations yielding to the final expressions of $S_{i j p q}^{\mathrm{NL}(\mathrm{dir})}$ and $S_{i j p q}^{\mathrm{NL} \text { (pol) }}$ are similar to the ones for $S_{i j}^{\mathrm{NL}(\mathrm{dir})}$ and $S_{i j}^{\mathrm{NL}(\mathrm{pol})}$, but somehow lengthier:

$$
\begin{aligned}
S_{i j p q}^{\mathrm{NL}(\mathrm{dir})}= & 2 \int_{\Delta_{k}} \theta_{k p q} \pi^{2} k^{2} p^{2} q\left(x y+z^{3}\right) \mathcal{E}_{0}^{\prime \prime}\left[\mathcal { E } _ { 0 } ^ { \prime } \left(H_{i j p q}^{(\mathrm{dir})^{\prime}}\left(35 z^{4}-30 z^{2}+3\right)\right.\right. \\
& \left.\left.+H_{i j p q}^{(\mathrm{dir})^{\prime \prime}}\left(35 y^{4}-30 y^{2}+3\right)\right)-\mathcal{E}_{0}\left(8 H_{i j p q}^{(\mathrm{dir})}+H_{i j p q}^{(\mathrm{dir})^{\prime \prime}}\left(35 y^{4}-30 y^{2}+3\right)\right)\right] \mathrm{d} p \mathrm{~d} q \\
& +\frac{2}{3} \int_{\Delta_{k}} \theta_{k p q} \pi^{2} k^{2} p^{2} q \mathcal{E}_{0}^{\prime \prime}\left[\left(x y+z^{3}\right)\left(1-y^{2}\right)\left(7 y^{2}-1\right)\left(\mathcal{E}_{0}^{\prime}-\mathcal{E}_{0}\right) H_{i j p q}^{(\mathrm{pol})^{\prime \prime}}\right. \\
+ & \left.z\left(1-z^{2}\right)^{2}\left(1-7 z^{2}\right) \mathcal{E}_{0}^{\prime} H_{i j p q}^{(\mathrm{pol})^{\prime}}\right] \mathrm{d} p \mathrm{~d} q, \\
S_{i j p q}^{\mathrm{NL}(\mathrm{pol})}= & 4 \int_{\Delta_{k}} \theta_{k p q} \pi^{2} k^{2} p^{2} q \mathcal{E}_{0}^{\prime \prime}\left[\left(x y+z^{3}\right)\left(\mathcal{E}_{0}^{\prime} H_{i j p q}^{(\mathrm{pol})^{\prime}}\left(1-6 z^{2}+7 z^{4}\right)-4 \mathcal{E}_{0} H_{i j p q}^{(\mathrm{pol})}\right)\right. \\
& +z\left(5-7 z^{2}\right)\left(y^{2}-z^{2}\right) \mathcal{E}_{0}^{\prime} H_{i j p q}^{(\mathrm{pol})^{\prime}}+\left(\mathcal{E}_{0}^{\prime}-\mathcal{E}_{0}\right) H_{i j p q}^{(\mathrm{pol})^{\prime \prime}} z\left(z^{2}-1\right)\left(1-6 y^{2}+7 y^{4}\right) \\
& \left.+x y\left(5-7 y^{2}\right)\left(1-z^{2}\right) \mathcal{E}_{0} H_{i j p q}^{(\mathrm{pol})^{\prime \prime}}\right] \mathrm{d} p \mathrm{~d} q+60 \int_{\Delta_{k}} \theta_{k p q} \pi^{2} k^{2} p^{2} q \mathcal{E}_{0}^{\prime \prime} z\left(1-z^{2}\right) \\
& \times\left[\left(1-8 y^{2}+7 y^{4}\right)\left(\mathcal{E}_{0}^{\prime}-\mathcal{E}_{0}\right) H_{i j p q}^{(\mathrm{dir})^{\prime \prime}}+\left(1-8 z^{2}+7 z^{4}\right) \mathcal{E}_{0}^{\prime} H_{i j p q}^{(\mathrm{dir})^{\prime}}\right] \mathrm{d} p \mathrm{~d} q, \quad(53)
\end{aligned}
$$

where $\Delta_{k}$ is the domain where $k, p$, and $q$ are the lengths of the sides of the triangle formed by the triad, and compact notations are used, $\mathcal{E}_{0}=E(k) /\left(4 \pi k^{2}\right), \mathcal{E}_{0}^{\prime}=E(p) /\left(4 \pi p^{2}\right)$, and $\mathcal{E}_{0}^{\prime \prime}=E(q) /\left(4 \pi q^{2}\right)$.

\subsection{Final spherically averaged equations}

As a consequence of the previous calculations, the evolution equations of the fourth-order anisotropic descriptors $E H_{i j p q}^{(\mathrm{dir})}$ and $E H_{i j p q}^{(\mathrm{pol})}$ read

$$
\begin{aligned}
& \left(\frac{\partial}{\partial t}+2 v k^{2}\right) E(k) H_{i j p q}^{(\mathrm{dir})}(k)=S_{i j p q}^{\mathrm{L}(\mathrm{dir} 2)}(k)+S_{i j p q}^{\mathrm{L}(\mathrm{dir} 4)}(k)+S_{i j p q}^{\mathrm{NL}(\mathrm{dir})}(k), \\
& \left(\frac{\partial}{\partial t}+2 v k^{2}\right) E(k) H_{i j p q}^{(\mathrm{pol})}(k)=S_{i j p q}^{\mathrm{L}(\mathrm{pol} 2)}(k)+S_{i j p q}^{\mathrm{L}(\mathrm{pol} 4)}(k)+S_{i j p q}^{\mathrm{NL}(\mathrm{pol})}(k) .
\end{aligned}
$$

Moreover, the original equations (19) and (20) of $E H_{i j}^{(\mathrm{dir})}$ and $E H_{i j}^{(\mathrm{pol})}$ derived in MCS are modified accordingly into

$$
\begin{aligned}
& \left(\frac{\partial}{\partial t}+2 v k^{2}\right) E(k, t) H_{i j}^{(\mathrm{dir})}(k)=S_{i j}^{\mathrm{L}(\operatorname{dir} 2)}(k)+S_{i j}^{\mathrm{L}(\mathrm{dir} 4)}(k)+S_{i j}^{\mathrm{NL}(\operatorname{dir})}(k), \\
& \left(\frac{\partial}{\partial t}+2 v k^{2}\right) E(k) H_{i j}^{(\mathrm{pol})}(k)=S_{i j}^{\mathrm{L}(\mathrm{pol} 2)}(k)+S_{i j}^{\mathrm{L}(\mathrm{pol} 4)}(k)+S_{i j}^{\mathrm{NL}(\mathrm{pol})}(k) .
\end{aligned}
$$




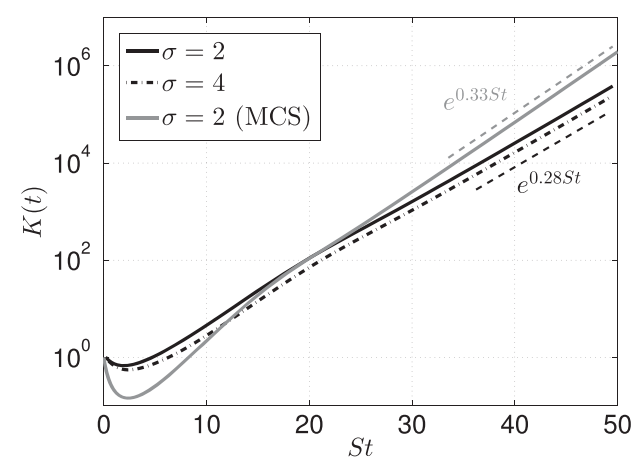

(a)

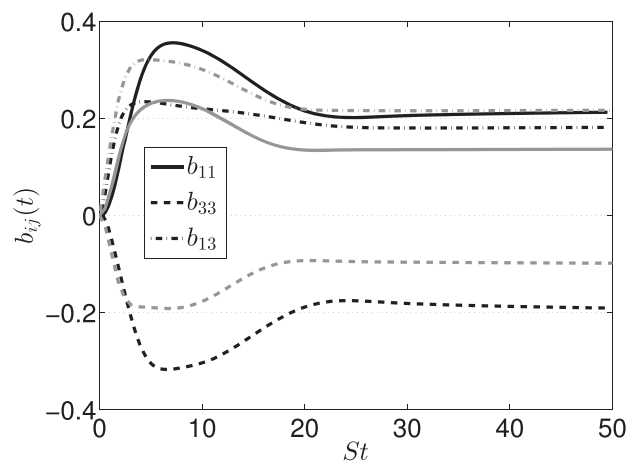

(b)

Figure 3. Effects of the fourth-order expansion on the growth of the kinetic energy $K(t)$ and the global anisotropy indicator $b_{i j}$. (a) $K(t)$ for $\sigma=2$ and $\sigma=4$. (b) $b_{i j}$ for $\sigma=2$. In both figures, the grey curves indicate a simulation without the fourth-order contributions for comparison.

At this point, it is crucial to understand that the retro-action of the fourth-order contributions on the second-order ones is uniquely done through the linear production terms $S_{i j}^{\mathrm{L}(\mathrm{dir} 4)}$ and $S_{i j}^{\mathrm{L}(\text { pol4) }}$, and that the impact of the second-order contributions on the fourth-order ones is uniquely done through the linear terms $S_{i j p q}^{\mathrm{L}(\mathrm{dir} 2)}$ and $S_{i j p q}^{\mathrm{L}(\mathrm{pol} 2)}$.

These four equations - the two new for $E H_{i j p q}^{(\mathrm{dir})}$ and $E H_{i j p q}^{(\mathrm{pol})}$ and the two adapted for $E H_{i j}^{(\mathrm{dir})}$ and $E H_{i j}^{(\mathrm{pol})}$ - represent the second important theoretical contribution of this work. For future reference, we call this approach the advanced anisotropic EDQNM modelling. In the next section, some numerical results using the fourth-order expansions are presented.

\subsection{Numerical results using the fourth-order expansion}

In this part, some numerical results are proposed to illustrate the interest of considering the fourth-order expansion of $\mathcal{E}$ and $Z$ in spherical harmonics. The numerical set-up is the same as in [3]: $k_{\min }=10^{-10} k_{L}(t=0)$ and $k_{\max }=10^{6} k_{\eta}$, where $k_{L}$ and $k_{\eta}=\left(\epsilon / \nu^{3}\right)^{1 / 4}$ are, respectively, the integral and Kolmogorov wavenumbers. For numerical integration of the five Lin equations of the advanced anisotropic EDQNM modelling, a logarithmic discretisation in wavenumbers is used, such that $k_{i+1}=r k_{i}$ with $r=10^{1 / f}, f=17$ points per decade, with a third-order implicit Runge-Kutta scheme for the viscous term. The initial Reynolds number based on the Taylor scale is approximately $\operatorname{Re}_{\lambda}(0) \simeq 10$, and an initial isotropic fully developed kinetic energy spectrum, given by [41], is chosen.

The main consequence of the fourth-order contributions is that the kinetic energy exponential growth rate is decreased in Figure 3(a), from $\gamma=0.33$ with the MCS model, to $\gamma=0.28$ with the present advanced anisotropic modelling. Even though the value of $\gamma$ remains rather large, the significant decrease by $15 \%$ with the fourth-order expansion of $\mathcal{E}$ and $Z$ perfectly proves that taking into account more spherical harmonics goes into the good direction, i.e. diminishes $\gamma$ towards smaller values, consistently with DNS.

The joint result is, in Figure 3(b), the decrease of $b_{13}$ from 0.215 to 0.18 , which is a noteworthy feature as well. One can further remark that on the contrary, the fourth-order 


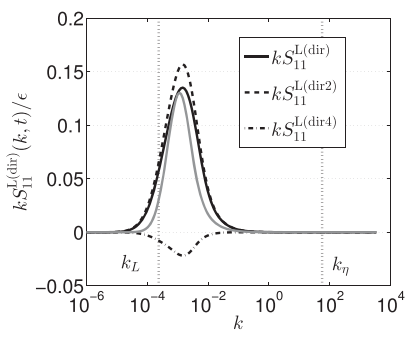

(a)

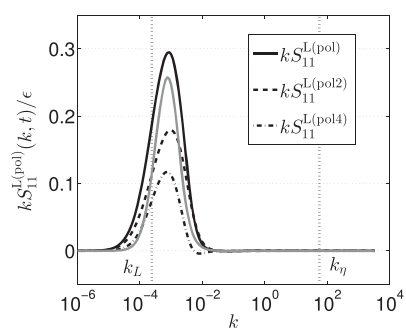

(d)

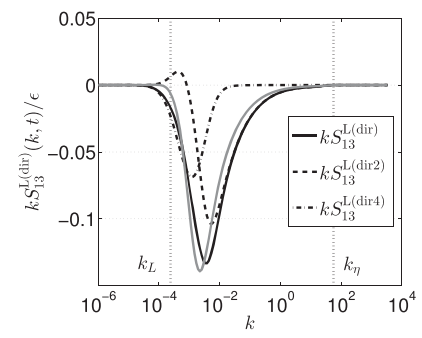

(b)

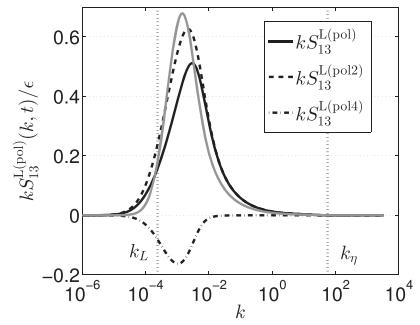

(e)

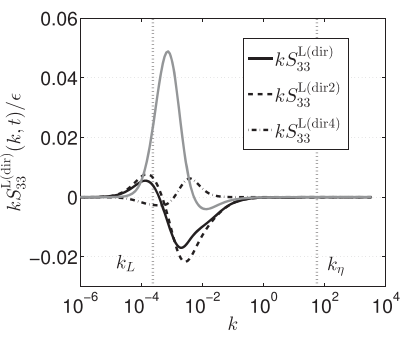

(c)

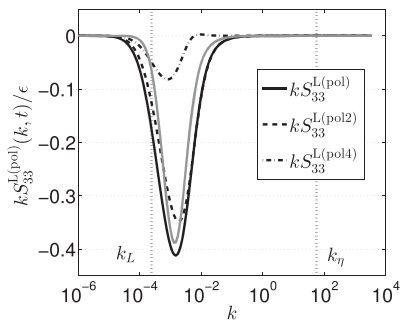

(f)

Figure 4. Effects of the fourth-order expansion on the linear terms of $E H_{i j}^{(\mathrm{dir})}$ and $E H_{i j}^{(\mathrm{pol})}$, for $\sigma=2$, at $R e_{\lambda}(S t=50)=9 \times 10^{3}$. The terms $S_{i j}^{\mathrm{L}(\mathrm{dir} 2)}$ and $S_{i j}^{\mathrm{L}(\text { pol2) }}$ contain the second-order contributions, and the terms $S_{i j}^{\mathrm{L}(\text { dir4) }}$ and $S_{i j}^{\mathrm{L}(\text { pol4) }}$ the fourth-order contributions. (a) $S_{11}^{\mathrm{L}(\text { dir) }}$, (b) $S_{13}^{\mathrm{L}(\text { dir) }}$, (c) $S_{33}^{\mathrm{L}(\text { dir) }}$, (d) $S_{11}^{\mathrm{L}(\text { pol) }}$, (e) $S_{13}^{\mathrm{L}(\mathrm{pol})}$, and (f) $S_{33}^{\mathrm{L}(\mathrm{pol})}$. In each case, the grey curves indicate simulations without the fourth-order contributions at $\operatorname{Re}_{\lambda}(S t=43)=9 \times 10^{3}$.

contributions increase $\left|b_{11}\right|$ and $\left|b_{33}\right|$, which is expected. Indeed, taking into account more harmonics reduces the loss of information due to the spherical integration by restoring part of the anisotropic angular information. As a consequence, the strong anisotropy of the shear flow between the streamwise and transverse directions is better captured.

To better understand the impact of the fourth-order contributions, we investigate the production terms in Figure 4, with the directional and polarisation parts of the streamwise, transverse and cross components in Saffman turbulence. In this figure, the black curves represent simulations with the fourth-order contributions, at $R e_{\lambda}=9 \times 10^{3}$. Whereas the grey curves indicate simulations with only the second-order expansion, as in MCS. Since the Reynolds number increases faster in the latter case, the results are presented at $S t=43$ where $R e_{\lambda} \simeq 9 \times 10^{3}$ as well.

One can remark that the effects are different for the directional and polarisation parts: indeed, the fourth-order contributions tend to decrease the intensity of the directional linear terms for the streamwise ()$_{11}$ and transverse ()$_{33}$ components, while increasing it for the ()$_{13}$ component. The opposite happens for the linear polarisation terms. In particular, the strongest difference is observed for the transverse directional transfer $S_{33}^{\mathrm{L} \text { (dir) }}$, which is positive without the fourth-order contributions, and becomes mostly negative with them.

As a conclusion for the fourth-order contributions, the main consequence is a $15 \%$ decrease of the kinetic energy exponential growth rate, namely from $\gamma=0.33$ to $\gamma=0.28$, which is quite significant, without rendering the numerical simulations more costly. It 
further justifies a posteriori the assumptions made to establish the fourth-order expansion of $\mathcal{E}$ and $Z$.

\section{Odd-order terms in the expansion of $Z$}

In this section, we discuss the possibility of odd-order terms in the spherical harmonics expansion of the polarisation anisotropy $Z$. Recall that because of the symmetry of $\hat{R}_{i j}$, the kinetic energy density $\mathcal{E}$ has only even-order contributions [12,23]. There are two reasons why we wish to further consider odd-order terms in the expansion of $Z$ : (i) Odd-order expansions could improve the modelling of the $2 \mathrm{i} Z \Omega_{\mathrm{CH}}$ term in the evolution equation (8) of $Z$; (ii) Recent results show that the main difference between the MCS model (and thus the truncated expansions of $\mathcal{E}$ and $Z$ ) with an exact treatment of linear terms lies in the polarisation anisotropy [15]. Unfortunately, these developments are not conclusive yet because they cannot be sustained numerically. Nevertheless, they are presented here for information purposes, and to underline that perhaps different methods than simple tensorial tools are required to model higher-order anisotropy features.

\subsection{Determination of the third-order expansion of $Z$}

Up to the fourth order, the expansion of $Z$ can be written as

$$
Z(\boldsymbol{k})=\frac{\mathcal{E}_{0}}{2}\left(5 H_{i j}^{(\mathrm{pol})}(k)+\mathrm{i} U_{i j k}^{(\mathrm{pol}) 3}(k) \alpha_{k}+\frac{21}{2} H_{i j p q}^{(\mathrm{pol})}(k) \alpha_{p} \alpha_{q}\right) N_{i}^{*}(\boldsymbol{k}) N_{j}^{*}(\boldsymbol{k}),
$$

where $U_{i j k}^{(\mathrm{pol}) 3}$ is a tensor which is assumed to verify, for simplicity reasons and consistency with previous developments, full symmetry under any change of indices, and to be zero when two indices are equal. Note that the expression of the third-order contribution differs from the one in [1] because of the imaginary number $i$. The latter is crucial, otherwise the third order of $Z$ never contributes in the equations. Two features are needed to prove this statement. First, one requires the following result when computing the polarisation part $\hat{R}_{i j}^{(\mathrm{pol})}=\mathfrak{R}\left[Z N_{i} N_{j}\right]$ :

$$
\begin{aligned}
N_{i} N_{j} N_{p}^{*} N_{q}^{*}= & {\left[P_{i p} P_{j q}+P_{i q} P_{j p}-P_{i j} P_{p q}\right] } \\
& -\frac{1}{2} \mathrm{i} \alpha_{a}\left[P_{j q} \epsilon_{i p a}+P_{i p} \epsilon_{j q a}+P_{i q} \epsilon_{j p a}+P_{j p} \epsilon_{i q a}\right],
\end{aligned}
$$

where $\epsilon_{i j k}$ is the Levi-Civita permutation tensor. The second one is that the spherical average of an odd number of normalised wavevectors $\alpha_{i}$ is zero [41]. Consequently, without the $\mathrm{i}, \mathfrak{R}\left[Z N_{i} N_{j}\right]$ has an odd number of $\alpha_{i}$ so that it vanishes with the spherical average. Therefore, the present expansion (58) of $Z$ corrects the Equation (3.15) of [1].

Then, as for the second- and fourth-order terms, one needs an operator which gives only the third-order contribution $U_{i j k}^{(\mathrm{pol}) 3}$ and erases the others. This operator reads $N_{i j k}=$ $\alpha_{k} N_{i} N_{j}+\alpha_{j} N_{i} N_{k}+\alpha_{i} N_{j} N_{k}$, and we further define $H_{i j k}^{(\mathrm{pol})}$ as

$$
2 E(k, t) H_{i j k}^{(\mathrm{pol})}(k, t)=\int_{S_{k}} \Im\left[Z(\boldsymbol{k}, t) N_{i j k}(\boldsymbol{k})\right] \mathrm{d}^{2} \boldsymbol{k}=\frac{2}{7} E(k, t) U_{i j k}^{(\mathrm{pol}) 3}(k, t) .
$$


The third-order expansion of $Z$ does not modify the spectral tensor $\phi_{i j}$ given in Equation (13), which is still expressed as a function of $H_{i j}^{(\mathrm{dir})}$ and $H_{i j}^{(\mathrm{pol})}$ only, and gives a new contribution in the modelled spectral Reynolds tensor $\hat{R}_{i j}$ :

$$
\hat{R}_{i j}^{\mathrm{z3}}(\boldsymbol{k}, t)=\frac{7}{2} \mathcal{E}_{0}(k, t) H_{p q l}^{(\mathrm{pol})}(k, t) \alpha_{l} \alpha_{n}\left(\epsilon_{i p n} P_{j q}(\boldsymbol{k})+\epsilon_{j q n} P_{i p}(\boldsymbol{k})\right) .
$$

Note that using some properties of the helical modes [27], the third-order expansion $2 Z=$ $7 \mathrm{i} H_{p q l}^{(\mathrm{pol})} \alpha_{l} N_{p}^{*} N_{q}^{*}$ can also be written $2 Z=7 H_{p q l}^{(\mathrm{pol})} \alpha_{l} \alpha_{r} \epsilon_{p r s} N_{q}^{*} N_{s}^{*}$. Now that the third-order expansion of $Z$ has been properly defined, one needs to compute the linear production terms and non-linear transfers associated to $E H_{i j k}^{(\mathrm{pol})}$, along with its retro-action on the evolution equations of $E H_{i j}^{(\mathrm{dir})}$ and $E H_{i j}^{(\mathrm{pol})}$. For simplicity, we do not consider in this work the linear coupling between the third and fourth orders.

Remark: It is worth noting that the third-order expansion of polarisation can be related to the stropholysis tensor [42] defined as

$$
\begin{aligned}
Q_{i j k}(t) & =\epsilon_{i p q} \int \alpha_{p} \alpha_{k} \hat{R}_{j q}(\boldsymbol{k}, t) \mathrm{d}^{3} \boldsymbol{k} \\
& =-\int \epsilon_{i j p} \alpha_{p} \alpha_{k} \mathcal{E}(\boldsymbol{k}, t) \mathrm{d}^{3} \boldsymbol{k}+\int \alpha_{k} \Im\left(Z(\boldsymbol{k}, t) N_{i}(\boldsymbol{k}) N_{j}(\boldsymbol{k})\right) \mathrm{d}^{3} \boldsymbol{k} .
\end{aligned}
$$

The expression of $Q_{i j k}$ as a function of the second-order anisotropic tensors can be found in [1]. Interestingly, the symmetric stropholysis $Q_{i j k}^{*}=\left(Q_{i j k}+Q_{i k j}+4\right.$ perm. $) / 6$ does not contain directional anisotropy (because $\epsilon_{i j p}=-\epsilon_{j i p}$ ) and depends only on polarisation. More specifically, only odd-order terms of the $Z$ expansion can contribute in $Q_{i j k}^{*}$, which further justifies the interest of odd-order contributions.

\subsection{Third-order productions terms}

In this part, and following the procedure detailed for the fourth-order contributions, we aim at determining the explicit expressions of the production terms linked to the thirdorder contributions in the expansion (58) of $Z$. As before, there are three different kinds of terms: the third-order contributions in the equations of $E H_{i j}^{(\mathrm{dir})}$ and $E H_{i j}^{(\mathrm{pol})}$ and in the equation of $E H_{i j k}^{(\mathrm{pol})}$, and finally the second-order contributions in the equations of $E H_{i j k}^{(\mathrm{pol})}$. There are no third-order contributions in the equation for $E$, and for simplicity reasons, we further discard the third-order contributions in the evolution equations of $E H_{i j p q}^{(\mathrm{dir})}$ and $E H_{i j p q}^{(\mathrm{pol})}$, and the fourth-order contributions in the equation of $E H_{i j k}^{(\mathrm{pol})}$.

First, the retro-action of the third-order expansion in the equations of $E H_{i j}^{(\mathrm{dir})}$ and $E H_{i j}^{(\text {pol) }}$ reads

$$
S_{i j}^{\mathrm{L}(\mathrm{dir} 3)}(k, t)=\frac{1}{6} A_{l n}^{+} E\left(\epsilon_{i l p} H_{j n p}^{(\mathrm{pol})}+\epsilon_{j l p} H_{i n p}^{(\mathrm{pol})}\right),
$$




$$
\begin{aligned}
S_{i j}^{\mathrm{L}(\mathrm{pol} 3)}(k, t)= & \frac{1}{3} A_{l n}^{-} E\left(\epsilon_{\ln p} H_{i j p}^{(\mathrm{pol})}-\epsilon_{i l p} H_{j n p}^{(\mathrm{pol})}-\epsilon_{j l p} H_{i n p}^{(\mathrm{pol})}\right) \\
& -\frac{1}{6} A_{l n}^{+}\left(\frac{\partial}{\partial k}\left[k E\left(\epsilon_{i l p} H_{j n p}^{(\mathrm{pol})}+\epsilon_{j l p} H_{i n p}^{(\mathrm{pol})}\right)\right]+E\left(\epsilon_{i l p} H_{j n p}^{(\mathrm{pol})}+\epsilon_{j l p} H_{i n p}^{(\mathrm{pol})}\right)\right) .
\end{aligned}
$$

Note that unlike $S_{i j}^{\mathrm{L}(\mathrm{dir} 4)}$ and $S_{i j}^{\mathrm{L}(\mathrm{pol} 4)}, S_{i j}^{\mathrm{L}(\text { pol3) }}$ depends on the antisymmetric part $A_{l n}^{-}$of the mean-velocity gradient matrix. Now, we define the linear polarisation term $S_{i j k}^{\mathrm{L}(\mathrm{pol})}$ of $E H_{i j k}^{(\mathrm{pol})}$ as

$$
S_{i j k}^{\mathrm{L}(\mathrm{pol})}=\frac{1}{4} \int_{S_{k}} \mathfrak{\Im}\left[\left(A_{l n} k_{l} \frac{\partial \hat{R}_{r s}}{\partial k_{n}}-M_{r n} \hat{R}_{n s}-M_{s n} \hat{R}_{n r}\right) N_{r}^{*} N_{s}^{*} N_{i j k}\right] \mathrm{d}^{2} \boldsymbol{k} .
$$

This production term is divided into two contributions resulting from the second- and third-order expansions. First, we determine the impact of the second-order contributions $E H_{i j}^{(\mathrm{dir})}$ and $E H_{i j}^{(\mathrm{pol})}$ on the linear term of $E H_{i j k}^{(\mathrm{pol})}$. For this purpose, we introduce two tracefree and symmetric operators, namely

$$
\begin{aligned}
\mathcal{H}_{i j k}^{2,+}\left[E H^{()}\right]= & 5 E\left[A_{i l}^{+}\left(\epsilon_{j l n} H_{n k}^{()}+\epsilon_{k l n} H_{n j}^{()}\right)+A_{j l}^{+}\left(\epsilon_{i l n} H_{n k}^{()}+\epsilon_{k l n} H_{n i}^{()}\right)\right. \\
+ & \left.A_{k l}^{+}\left(\epsilon_{i l n} H_{n j}^{()}+\epsilon_{j l n} H_{n i}^{()}\right)\right]-2 E A_{l n}^{+} H_{n p}^{()} E\left[\delta_{i j} \epsilon_{k l p}+\delta_{i k} \epsilon_{j l p}+\delta_{j k} \epsilon_{i l p}\right], \\
\mathcal{H}_{i j k}^{2,-}\left[E H^{()}\right]= & 5 E\left[A_{i l}^{-}\left(\epsilon_{j l n} H_{n k}^{()}+\epsilon_{k l n} H_{n j}^{()}\right)+A_{j l}^{-}\left(\epsilon_{i l n} H_{n k}^{()}+\epsilon_{k l n} H_{n i}^{()}\right)\right. \\
& \left.+A_{k l}^{-}\left(\epsilon_{i l n} H_{n j}^{()}+\epsilon_{j l n} H_{n i}^{()}\right)-A_{l n}^{-}\left(\epsilon_{i l n} H_{j k}^{()}+\epsilon_{j l n} H_{i k}^{()}+\epsilon_{k l n} H_{i j}^{()}\right)\right] \\
& +2 E A_{l n}^{-} H_{n p}^{()} E\left[\delta_{i j} \epsilon_{k l p}+\delta_{i k} \epsilon_{j l p}+\delta_{j k} \epsilon_{i l p}\right] .
\end{aligned}
$$

After some algebra, one gets

$$
\begin{aligned}
S_{i j k}^{\mathrm{L}(\mathrm{pol} 2)}= & -\frac{1}{7} \mathcal{H}_{i j k}^{(2,+)}\left[E H^{(\mathrm{dir})}\right]+\frac{1}{21}\left[\mathcal{H}_{i j k}^{(2,-)}\left[E H^{(\mathrm{pol})}\right]-\mathcal{H}_{i j k}^{(2,+)}\left[E H^{(\mathrm{pol})}\right]\right] \\
& +\frac{1}{42} \mathcal{H}_{i j k}^{(2,+)}\left[\partial_{k}\left(k E H^{(\mathrm{pol})}\right)\right] .
\end{aligned}
$$

Now, we proceed similarly to determine the linear term of $E H_{i j k}^{(\mathrm{pol})}$ as a function of the third-order contributions. Eventually, one gets

$$
S_{i j k}^{\mathrm{L}(\mathrm{pol} 3)}=\frac{1}{3}\left[A_{i l}^{-} H_{j l k}^{(\mathrm{pol})}+A_{j l}^{-} H_{i l k}^{(\mathrm{pol})}+A_{k l}^{-} H_{i j l}^{(\mathrm{pol})}\right],
$$

where notably the symmetric part of the mean-velocity gradient matrix does not intervene. Finally, the total third-order polarisation transfer is $S_{i j k}^{\mathrm{L}(\mathrm{pol})}=S_{i j k}^{\mathrm{L}(\text { pol2) }}+S_{i j k}^{\mathrm{L}(\text { pol3)}}$. 


\subsection{Third-order non-linear transfer}

The third-order polarisation non-linear transfer is defined as

$$
S_{i j k}^{\mathrm{NL}(\mathrm{pol})}(k, t)=\frac{1}{2} \int_{S_{k}} \Im\left(T_{Z}(\boldsymbol{k}, t) N_{i j k}(\boldsymbol{k})\right) \mathrm{d}^{2} \boldsymbol{k} .
$$

For the sake of simplicity, quadratic anisotropic contributions are still discarded, as for the second and fourth orders: therefore, only the third-order terms contribute in $S_{i j k}^{\mathrm{NL}(\mathrm{pol})}$. Furthermore, because $H_{i j k}^{(\mathrm{pol})}$ is symmetric and trace-free, it follows that third-order expansions vanish in $S_{i j}^{\mathrm{NL}(\mathrm{dir})}$ and $S_{i j}^{\mathrm{NL}(\mathrm{pol})}$. Then, injecting the third-order expansion into the expression (17) of $T_{Z}$ gives the spherically averaged non-linear polarisation transfer

$$
\begin{aligned}
S_{i j k}^{\mathrm{NL}(\mathrm{pol})}= & 4 \pi^{2} \int_{\Delta_{k}} \theta_{k p q} k^{2} p^{2} q \mathcal{E}_{0}^{\prime \prime}\left[-4\left(x y+z^{3}\right) \mathcal{E}_{0} H_{i j k}^{(\mathrm{pol})}\right. \\
& +H_{i j k}^{(\mathrm{pol})^{\prime \prime}}\left(1-z^{2}\right)\left(2 z\left(1-2 y^{2}\right)\left(\mathcal{E}_{0}^{\prime}-\mathcal{E}_{0}\right)+x y\left(1-3 y^{2}\right) \mathcal{E}_{0}\right) \\
& \left.+H_{i j k}^{(\mathrm{pol})^{\prime}} \mathcal{E}_{0}^{\prime}\left(2\left(x y+z^{3}\right)\left(2 z^{2}-1\right)-z\left(3 z^{2}-1\right)\left(y^{2}-z^{2}\right)\right)\right] \mathrm{d} p \mathrm{~d} q .
\end{aligned}
$$

\subsection{Final spherically averaged equations}

The evolution equation of the third-order anisotropic descriptor $E H_{i j k}^{(\mathrm{pol})}$ reads

$$
\left(\frac{\partial}{\partial t}+2 v k^{2}\right) E(k) H_{i j k}^{(\mathrm{pol})}(k)=S_{i j k}^{\mathrm{L}(\mathrm{pol} 2)}(k)+S_{i j k}^{\mathrm{L}(\mathrm{pol} 3)}(k)+S_{i j k}^{\mathrm{NL}(\mathrm{pol})}(k) .
$$

Moreover, the original equations (19) and (20) of $E H_{i j}^{(\mathrm{dir})}$ and $E H_{i j}^{(\mathrm{pol})}$ derived in MCS are modified accordingly into

$$
\begin{aligned}
& \left(\frac{\partial}{\partial t}+2 \nu k^{2}\right) E(k, t) H_{i j}^{(\mathrm{dir})}(k)=S_{i j}^{\mathrm{L}(\mathrm{dir} 2)}(k)+S_{i j}^{\mathrm{L}(\mathrm{dir} 3)}(k)+S_{i j}^{\mathrm{NL}(\mathrm{dir})}(k), \\
& \left(\frac{\partial}{\partial t}+2 \nu k^{2}\right) E(k) H_{i j}^{(\mathrm{pol})}(k)=S_{i j}^{\mathrm{L}(\mathrm{pol} 2)}(k)+S_{i j}^{\mathrm{L}(\mathrm{pol} 3)}(k)+S_{i j}^{\mathrm{NL}(\mathrm{pol})}(k) .
\end{aligned}
$$

Note that the retro-action of the third-order contributions on the second-order ones is uniquely done through the linear transfers $S_{i j}^{\mathrm{L}(\mathrm{dir} 3)}$ and $S_{i j}^{\mathrm{L}(\mathrm{pol3})}$, and that the impact of the second-order contributions on the third-order ones is uniquely done through the linear transfers $S_{i j k}^{\mathrm{L}(\mathrm{dir} 2)}$ and $S_{i j k}^{\mathrm{L}(\mathrm{pol} 2)}$. This is completely similar to what was done for the fourth order.

These developments for the third order of the polarisation anisotropy $Z$ are not conclusive yet because the numerical code cannot handle satisfactorily the corresponding equations (72), (73), and (74). This is very likely because of the assumptions we made to define $H_{i j l}^{(\mathrm{pol})}$, namely the full symmetry between indices. Similar rules were nevertheless successfully used previously for $H_{i j p q}^{(\mathrm{dir})}$ and $H_{i j p q}^{(\mathrm{pol})}$ in Section 3. This suggests that more complex features should be taken into account for odd-order terms with more sophisticated tools. 


\section{Conclusions, perspectives, and summary}

This work focuses on the spectral modelling of shear flows using an adapted eddy-damped quasi-normal Markovian (EDQNM) closure for homogeneous anisotropic turbulence developed by Mons, Cambon, and Sagaut (MCS) [1] and further applied in [3]. The present paper should be considered as the continuity of the two previous publications. The main objectives were twofold: (i) to explain why in MCS the exponential growth rate of kinetic energy $\gamma$ is numerically independent of the large scales initial conditions, namely the infrared slope $\sigma$ of the kinetic energy spectrum $E\left(k<k_{L}\right) \sim k^{\sigma}$. And (ii) to show that $\gamma$ can actually be reduced by improving the modelling of anisotropy. Two secondary aspects were analysed as well: (iii) to quantify the effects of quadratic anisotropic contributions in the non-linear transfers, an assumption which was not verified in [1,3]. And (iv) to address the modelling of odd-order terms in the polarisation anisotropy expansion, a work for now inconclusive but still ongoing.

For the first point, it was erroneously thought by the authors in [3] that such an independence could be a consequence of the spherical average. It has been shown here analytically in Section 2 using self-similarity arguments that $\gamma$ is almost independent of $\sigma$ because the most amplified eigenvalue of the linear operator also depends on $\sigma$. This balances the $\sigma$-dependence of the self-similar expression of $E(k, t)$ at large scales. Interestingly, the anisotropic EDQNM model recovers well for unstably stratified homogeneous turbulence (USHT) the strong dependence of the kinetic energy exponential growth rate with the infrared slope $\sigma$ [4]: this is because the most amplified eigenvalue of the USHT linear operator does not depend on $\sigma$. This analysis consequently shows nicely that the mechanisms at the origin of the exponential growth in shear and USHT are intrinsically different. This is the first important finding of this work.

For the second point, we aimed at improving the MCS modelling for pure shear flows, whose main issue is that the kinetic energy exponential growth rate $\gamma$ is larger than common value reported in DNS and experiments [3]. Consequently, the objective here was to show that $\gamma$ could be reduced by better capturing the complex distribution of anisotropy. This was done by computing analytically the fourth-order terms in the spherical harmonics expansion of the kinetic energy density $\mathcal{E}$ and polarisation $Z$ (in MCS the truncation is at the second order). These developments led to the definitions of two new spherically averaged anisotropic descriptors $H_{i j p q}^{(\mathrm{dir})}$ and $H_{i j p q}^{(\mathrm{pol})}$ whose evolution equations are similar to the ones of the second-order descriptors $H_{i j}^{(\mathrm{dir})}$ and $H_{i j}^{(\mathrm{pol})}$. For simplicity reasons, we chose some symmetry rules for $H_{i j p q}^{(\mathrm{pol})}$ whose consequences are that the coupling between the second-order and fourth-order equations is purely linear, i.e. through the production terms.

Numerically, this advanced modelling for the anisotropy is satisfactory because it decreases the exponential growth rate of kinetic energy from $\gamma=0.33$ (MCS) to $\gamma=0.28$, without increasing the computational time. Conjointly, the cross-component of the global anisotropy indicator goes down to $\left|b_{13}\right|=0.18$. Hence, we indeed showed that taking into account more spherical harmonics in the expansion reduces the kinetic energy exponential growth rate by better capturing the anisotropy distribution. This is the second theoretical contribution of this work.

Regarding the third point, quadratic anisotropic contributions were explicitly presented in Appendix 2 and were shown to be numerically negligible in Section 2.5. This notably 
shows that to improve the description of anisotropy, it is more efficient to refine the linear production terms than the non-linear ones: a useful trail for future works.

Finally, the fourth and last aspect of this work was to address the modelling of oddorder terms in the expansion of polarisation anisotropy $Z$. All the analytical calculations were done but are inconclusive in terms of numerical simulations. This is probably due to assumptions we made regarding the symmetry properties of the third-order polarisation tensor $H_{i j k}^{(\mathrm{pol})}$. It was nevertheless shown that odd-order contributions of $Z$ can be related to the stropholysis [42] and as such deserve further investigations.

As perspectives, it could be of great theoretical interest to determine, or at least estimate, at which order one should perform the expansion into spherical harmonics to properly capture all the anisotropic features of a given homogeneous anisotropic turbulent flow. This task could be rather complex, and even if it is out of the scope of the present work, the authors would like to emphasis some aspects.

First, one would have to solve numerically the linear production terms of Equations (7) and (8) and to project $\mathcal{E}$ and $Z$ on an appropriate basis of deviatoric tensors such as $H_{i j}^{()}$, $H_{i j k}^{()}, H_{i j p q}^{()}, \ldots .:$ in particular, this would require to determine explicitly the higher-order projectors which may be extremely tedious given the complexity of the algebra involved already at the fourth-order.

Second, it is very likely that this required number of spherical harmonics would depend on both the mean-velocity gradient matrix $A_{i j}$ and the accumulated anisotropy $S t$. Indeed,

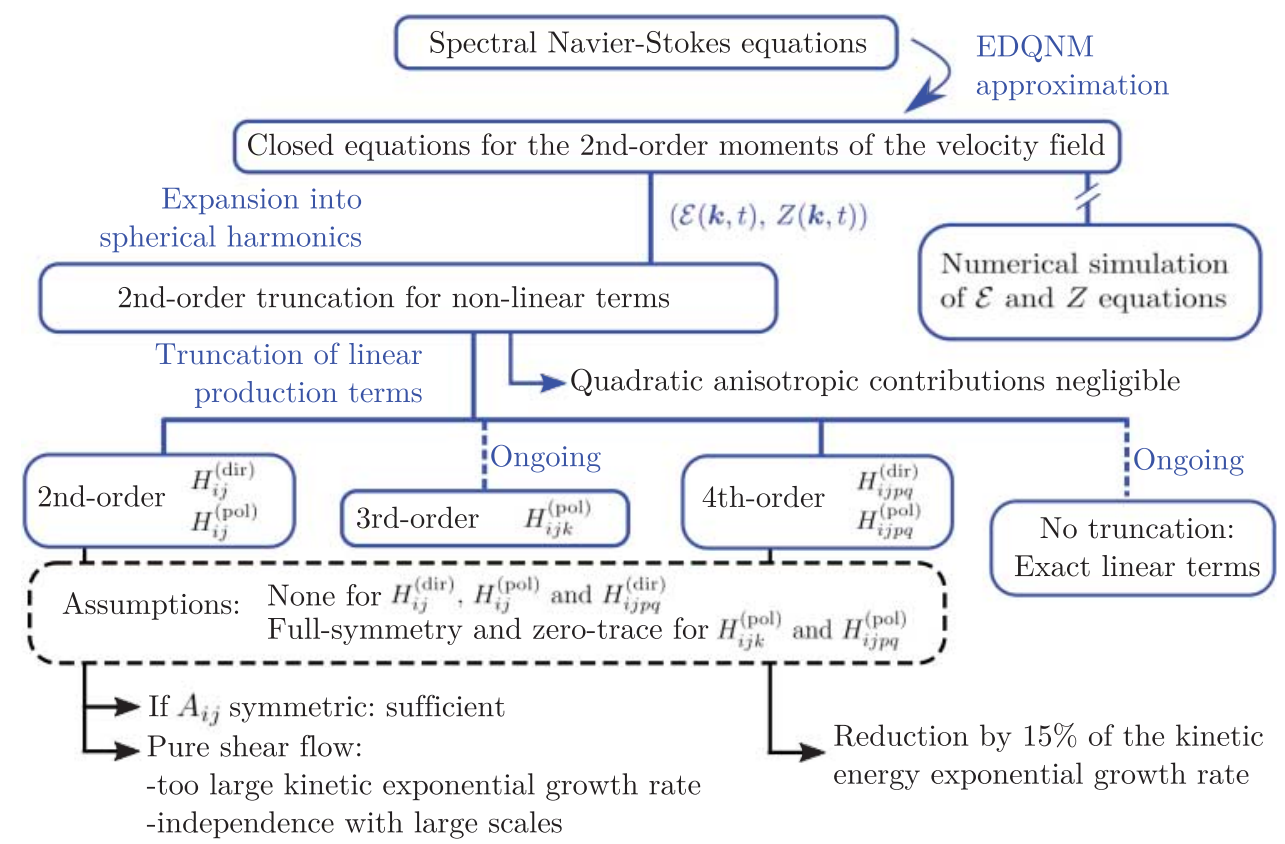

Figure 5. Diagram presenting the methodology of the modelling and summarising the different levels of assumptions, the various achievements, and the ongoing studies as well. The 'path' toward the numerical simulations of the full $\mathcal{E}$ - $Z$ equations with non-linear terms closed by EDQNM is broken since this would be almost as costly as DNS in terms of numerical resources. 
for the former, it appeared in [1,3] that the MCS model is accurate enough when $A_{i j}$ is symmetric, such as in an axisymmetric expansion or contraction, or in a plane distortion, so that the second-order expansion is sufficient. However, in shear flows, the fact that $A_{i j}$ is not symmetric anymore seems to imply that more harmonics are needed. Furthermore, in shear flows, the MCS model works fine at small St (see the comparison with rapid distortion theory in [1]), which means that the number of spherical harmonics needed would increase with St. Consequently, these two aspects make the task of predicting the order at which one should truncate the expansion of $\mathcal{E}$ and $Z$ into spherical harmonics quite intricate. An alternative solution could be to combine the anisotropic EDQNM modelling for the nonlinear transfers with an exact treatment of the linear production terms, which is currently the task of Zhu et al. in [15].

Finally, we propose in Figure 5 a synthetic view of the work accomplished so far, the various achievements, the methodology, the assumptions made, and the remaining objectives as well. Notably, Figure 5 shows that the short-term objective of our work is to improve the modelling of linear production terms with in particular the third-order expansion of $Z$. This will provide the turbulence community a model which can handle accurately secondorder moments statistics in shear flows, with very few numerical resources and at large Reynolds numbers.

\section{References}

[1] Mons V, Cambon C, Sagaut P. A spectral model for homogeneous shear-driven anisotropic turbulence in terms of spherically-averaged descriptors. J Fluid Mech. 2016;788:147-182.

[2] Briard A, Gomez T, Cambon C. Spectral modelling for passive scalar dynamics in homogeneous anisotropic turbulence. J Fluid Mech. 2016;799:159-199.

[3] Briard A, Gomez T, Mons V, et al. Decay and growth laws in homogeneous shear turbulence. J Turbul. 2016;17:699-726.

[4] Briard A, Iyer M, Gomez T. Anisotropic spectral modelling for unstably stratified homogeneous turbulence. Phys Rev Fluids. 2017;2:044604.

[5] Burlot A, Gréa BJ, Godeferd FS, et al. Spectral modelling of high Reynolds number unstably stratified homogeneous turbulence. J Fluid Mech. 2015;765:17-44.

[6] Briard A, Gomez T. Dynamics of helicity in homogeneous skew-isotropic turbulence. J Fluid Mech. 2017;821:539-581.

[7] Cambon C, Mons V, Gréa BJ, et al. Anisotropic triadic closures for shear-driven and buoyancydriven turbulent flows. Comp Fluids. 2017;151:73-84.

[8] Cambon C, Danaila L, Godeferd FS, et al. Third-order statistics and the dynamics of strongly anisotropic turbulent flows. J Turbul. 2013;14:121-160.

[9] Gréa BJ, Burlot A, Godeferd FS, et al. Dynamics and structure of unstably stratified homogeneous turbulence. J Turbul. 2016;17:651-663.

[10] Isaza JC, Collins LR. On the asymptotic behaviour of large-scale turbulence in homogeneous shear flow. J Fluid Mech. 2009;637:213-239.

[11] Sukheswalla P, Vaithianathan T, Collins LR. Simulation of homogeneous turbulent shear flows at higher Reynolds numbers: numerical challenges and a remedy. J Turbul. 2013;14:60-97. 
[12] Cambon C, Rubinstein R. Anisotropic developments for homogeneous shear flows. Phys Fluids. 2006;18:085106.

[13] Cambon C, Jacquin L. Spectral approach to non-isotropic turbulence subjected to rotation. J Fluid Mech. 1989;202:295-317.

[14] Briard A, Gomez T. Prandtl number effects in decaying homogeneous isotropic turbulence with a mean scalar gradient. J Turbul. 2017;18:418-442.

[15] Zhu Y, Cambon C, Godeferd FS. Rotating shear-driven turbulent flows: towards a spectral model with angle-dependent linear interactions. In: Shao JF, Mompean G, Markiewicz E, editors. Proceedings of the 23rd Congrès Francais de Mécanique; 2017. Available from: https:// cfm2017.sciencesconf.org/135227

[16] Casciola CM, Gualtieri P, Benzi R, et al. Scale-by-scale budget and similarity laws for shear turbulence. J Fluid Mech. 2003;476:105-114.

[17] Gualtieri P, Casciola CM, Benzi GAR, et al. Scaling laws and intermittency in homogeneous shear flow. Phys Fluids. 2002;14:583-596.

[18] Biferale L, Vergassola M. Isotropy vs anisotropy in small-scale turbulence. Phys Fluids. 2001;13:2139-2141.

[19] Schumacher J, Sreenivasan KR, Yeung PK. Derivative moments in turbulent shear flows. Phys Fluids. 2003;15:84.

[20] Pumir A. Turbulence in homogeneous shear flows. Phys Fluids. 1996;8:3112-3127.

[21] Garg S, Warhaft Z. On the small scale structure of simple shear flow. Phys Fluids. 1998;10:662-673.

[22] Shen $X$, Warhaft $Z$. The anisotropy of the small scale structure in high Reynolds number $\left(R_{\lambda} \sim\right.$ 1000) turbulent shear flow. Phys Fluids. 2000;12:2976-2989.

[23] Rubinstein R, Kurien S, Cambon C. Scalar and tensor spherical harmonics expansion of the velocity correlation in homogeneous anisotropic turbulence. J Turbul. 2015;16:1058-1075.

[24] Biferale L, Procaccia I. Anisotropy in turbulent flows and in turbulent transport. Phys Rep. 2005;414:43-164.

[25] Clark TT, Kurien S, Rubinstein R. Generation of anisotropy in turbulent flows subjected to rapid distortion. Phys Rev E. 2018;97:013112.

[26] Waleffe F. The nature of triad interactions in homogeneous turbulence. Phys Fluids. 1992;4:350-363.

[27] Sagaut P, Cambon C. Homogeneous turbulence dynamics. 1st ed. New York: Cambridge University Press; 2008.

[28] Lesieur M. Turbulence in fluids. 4th ed. Dordrecht: Springer; 2008 (Fluid mechanics and its applications; vol. 84).

[29] Eyink GL, Thomson DJ. Free decay of turbulence and breakdown of self-similarity. Phys Fluids. 2000;12:477-479.

[30] Clark TT, Zemach C. A spectral model applied to homogeneous turbulence. Phys Fluids. 1995;7:1674-1694.

[31] Tavoularis S. Asymptotic laws for transversely homogeneous turbulent shear flows. Phys Fluids. 1985;28:999-1001.

[32] George WK. The decay of homogeneous isotropic turbulence. Phys Fluids A. 1992;7:1492-1509.

[33] Tavoularis S, Corrsin S. Experiments in nearly homogenous turbulent shear flow with a uniform mean temperature gradient. Part 1. J Fluid Mech. 1981;104:311-347.

[34] Tavoularis S, Karnik U. Further experiments on the evolution of turbulent stresses and scales in uniformly sheared turbulence. J Fluid Mech. 1989;204:457-478.

[35] Souza FAD, Nguyen VD, Tavoularis S. The structure of highly sheared turbulence. J Fluid Mech. 1995;303:155-167.

[36] Brethouwer G. The effect of rotation on rapidly sheared homogeneous turbulence and passive scalar transport. Linear theory and direct numerical simulation. J Fluid Mech. 2005;542:305-342.

[37] Pumir A, Shraiman BI. Persistent small scale anisotropy in homogeneous shear flows. Phys Rev Lett. 1995;75:3114-3117. 
[38] Soulard O, Griffond J, Gréa BJ. Large-scale analysis of self-similar unstably stratified homogeneous turbulence. Phys Fluids. 2014;26:015110.

[39] Soulard O, Gréa BJ. Influence of zero-modes on the inertial-range anisotropy of RayleighTaylor and unstably stratified homogeneous turbulence. Phys Rev Fluids. 2017;2:074603.

[40] Briard A. Modelling of transport in homogeneous turbulence [thesis]. UPMC - Université Paris 6 Pierre et Marie Curie, Institut Jean le Rond d’Alembert; 2017.

[41] Pope SB. Turbulent flows. Cambridge: Cambridge University Press; 2000.

[42] Kassinos SC, Reynolds WC, Rogers MM. One-point turbulence structure tensors. J Fluid Mech. 2001;428:213-248.

\section{Appendices}

\section{Appendix 1. Linear and non-linear transfers for the second-order expansion}

In this appendix, the explicit expressions of the spherically averaged transfer terms are given. The non-linear ones are

$$
\begin{aligned}
& S^{\mathrm{NL}(\mathrm{iso})}=\int_{S_{k}} T_{\mathcal{E}} \mathrm{d}^{2} \boldsymbol{k}=16 \pi^{2} \int_{\Delta_{k}} \theta_{k p q} k^{2} p^{2} q\left(x y+z^{3}\right) \mathcal{E}_{0}^{\prime \prime}\left(\mathcal{E}_{0}^{\prime}-\mathcal{E}_{0}\right) \mathrm{d} p \mathrm{~d} q \\
& S_{i j}^{\mathrm{NL}(\mathrm{dir})}= \frac{1}{2} \int_{S_{k}} T_{\mathcal{E}} P_{i j} \mathrm{~d}^{2} \boldsymbol{k}-\frac{\delta_{i j}}{3} S^{\mathrm{NL}(\mathrm{iso})}=4 \pi^{2} \int_{\Delta_{k}} \theta_{k p q} k^{2} p^{2} q \mathcal{E}_{0}^{\prime \prime}\left[z\left(1-z^{2}\right)^{2} \mathcal{E}_{0}^{\prime} H_{i j}^{(\mathrm{pol})^{\prime}}\right. \\
&\left.+\left(y^{2}-1\right)\left(x y+z^{3}\right)\left(\mathcal{E}_{0}^{\prime}-\mathcal{E}_{0}\right) H_{i j}^{(\mathrm{pol})^{\prime \prime}}\right] \mathrm{d} p \mathrm{~d} q+8 \pi^{2} \int_{\Delta_{k}} \theta_{k p q} k^{2} p^{2} q\left(x y+z^{3}\right) \mathcal{E}_{0}^{\prime \prime} \\
& \times\left[\left(3 y^{2}-1\right)\left(\mathcal{E}_{0}^{\prime}-\mathcal{E}_{0}\right) H_{i j}^{(\mathrm{dir})^{\prime \prime}}+\left(3 z^{2}-1\right) \mathcal{E}_{0}^{\prime} H_{i j}^{(\mathrm{dir})^{\prime}}-2 \mathcal{E}_{0} H_{i j}^{(\mathrm{dir})}\right] \mathrm{d} p \mathrm{~d} q, \\
& S_{i j}^{\mathrm{NL}(\mathrm{pol})}= \frac{1}{2} \int_{S_{k}} \Re\left(T_{Z} N_{i} N_{j}\right) \mathrm{d}^{2} \boldsymbol{k}=4 \pi^{2} \int_{\Delta_{k}} \theta_{k p q} k^{2} p^{2} q \mathcal{E}_{0}^{\prime \prime}\left[2 z\left(z^{2}-y^{2}\right) \mathcal{E}_{0}^{\prime} H_{i j}^{(\mathrm{pol})^{\prime}}\right. \\
&+\left(x y+z^{3}\right)\left(\left(1+z^{2}\right) \mathcal{E}_{0}^{\prime} H_{i j}^{(\mathrm{pol})^{\prime}}-4 \mathcal{E}_{0} H_{i j}^{(\mathrm{pol})}\right)+z\left(z^{2}-1\right)\left(1+y^{2}\right)\left(\mathcal{E}_{0}^{\prime}-\mathcal{E}_{0}\right) H_{i j}^{(\mathrm{pol})^{\prime \prime}} \\
&+\left.2 x y\left(z^{2}-1\right) \mathcal{E}_{0} H_{i j}^{(\mathrm{pol})^{\prime \prime}}\right] \mathrm{d} p \mathrm{~d} q+24 \pi^{2} \int_{\Delta_{k}} \theta_{k p q^{2}} k^{2} p^{2} q z\left(z^{2}-1\right) \mathcal{E}_{0}^{\prime \prime} \\
& \times\left[\left(y^{2}-1\right)\left(\mathcal{E}_{0}^{\prime}-\mathcal{E}_{0}\right) H_{i j}^{(\mathrm{dir})^{\prime \prime}}+\left(z^{2}-1\right) \mathcal{E}_{0}^{\prime} H_{i j}^{(\mathrm{dir})^{\prime}}\right] \mathrm{d} p \mathrm{~d} q .
\end{aligned}
$$

From the total non-linear transfer, a return to isotropy (RTI) term can be extracted

$$
\begin{aligned}
S_{i j}^{(\mathrm{RTI})}(k, t)= & \int_{\Delta_{k}} \theta_{k p q} 16 \pi^{2} k^{2} p^{2} q(x+y z) \mathcal{E}_{0}^{\prime \prime}\left[\mathcal { E } _ { 0 } ^ { \prime } \left(y\left(z^{2}-y^{2}\right)\left(6 H_{i j}^{(\mathrm{dir})^{\prime \prime}}+H_{i j}^{(\mathrm{pol})^{\prime \prime}}\right)\right.\right. \\
& \left.\left.-(x z+y) H_{i j}^{(\mathrm{pol})^{\prime \prime}}\right)-y\left(z^{2}-x^{2}\right) \mathcal{E}_{0}\left(6 H_{i j}^{(\mathrm{dir})^{\prime \prime}}+H_{i j}^{(\mathrm{pol})^{\prime \prime}}\right)\right] \mathrm{d} p \mathrm{~d} q .
\end{aligned}
$$

All the non-linear transfers involve the characteristic time $\theta_{k p q}$ of the triple correlations, which appears within the EDQNM approximation and is defined as

$$
\theta_{k p q}=\frac{1-\mathrm{e}^{-\mu_{k p q} t}}{\mu_{k p q}}, \quad \mu_{k p q}=v\left(k^{2}+p^{2}+q^{2}\right)+\mu(k, t)+\mu(p, t)+\mu(q, t),
$$

where $\mu(k, t)$ is the (isotropic) eddy-damping factor [28]

$$
\mu(k, t)=A \sqrt{\int_{0}^{k} x^{2} E(x, t) \mathrm{d} x}
$$


and $A=0.36$. The production terms are, with $A_{i j}$ being the mean-velocity gradient matrix where ()$^{+}$ and ()$^{-}$denote the symmetric and antisymmetric parts,

$$
\begin{aligned}
S^{\mathrm{L}(\mathrm{iso})}= & \frac{1}{2} \int_{S_{k}}\left(A_{l n} k_{l} \frac{\partial \hat{R}_{i i}}{\partial k_{n}}-2 M_{i n} \hat{R}_{n i}\right) \mathrm{d}^{2} \boldsymbol{k} \\
= & -2 A_{l m}^{+}\left(\frac{\partial}{\partial k}\left(k E H_{l m}^{(\mathrm{dir})}\right)+E\left(H_{l m}^{(\mathrm{dir})}+H_{l m}^{(\mathrm{pol})}\right)\right) \\
S_{i j}^{\mathrm{L}(\mathrm{dir})}= & \frac{1}{4} \int_{S_{k}}\left(A_{l n} k_{l} \frac{\partial \hat{R}_{m m}}{\partial k_{n}}-2 M_{m n} \hat{R}_{n m}\right) P_{i j} \mathrm{~d}^{2} \boldsymbol{k}-\frac{\delta_{i j}}{3} S^{\mathrm{L}(\mathrm{iso})} \\
= & \frac{2}{15} A_{i j}^{+} E-\frac{2}{7} E\left(A_{l j}^{+} H_{i l}^{(\mathrm{pol})}+A_{l i}^{+} H_{j l}^{(\mathrm{pol})}-\frac{2}{3} A_{l n}^{+} \delta_{i j} H_{l n}^{(\mathrm{pol})}\right)-\frac{1}{15} A_{i j}^{+} \frac{\partial(k E)}{\partial k} \\
& +\frac{2}{7}\left(A_{i l}^{+} \frac{\partial}{\partial k}\left(k E H_{j l}^{(\mathrm{dir})}\right)+A_{j l}^{+} \frac{\partial}{\partial k}\left(k E H_{i l}^{(\mathrm{dir})}\right)-\frac{2}{3} A_{l m}^{+} \delta_{i j} \frac{\partial}{\partial k}\left(k E H_{l m}^{(\mathrm{dir})}\right)\right) \\
& -\frac{1}{7} E\left(A_{j l}^{+} H_{i l}^{(\mathrm{dir})}+A_{i l}^{+} H_{j l}^{(\mathrm{dir})}-\frac{2}{3} A_{l m}^{+} H_{l m}^{(\mathrm{dir})} \delta_{i j}\right)+E\left(A_{j n}^{-} H_{n i}^{(\mathrm{dir})}+A_{i n}^{-} H_{n j}^{(\mathrm{dir})}\right), \\
S_{i j}^{\mathrm{L}(\mathrm{pol})}= & \frac{1}{4} \int_{S_{k}} \Re\left[\left(A_{l n} k_{l} \frac{\partial \hat{R}_{r s}}{\partial k_{n}}-M_{r n} \hat{R}_{n s}-M_{s n} \hat{R}_{n r}\right) N_{r}^{*} N_{s}^{*} N_{i} N_{j}\right] \mathrm{d}^{2} \boldsymbol{k} \\
= & -\frac{2}{5} A_{i j}^{+} E-\frac{12}{7} E\left(A_{j l}^{+} H_{i l}^{(\mathrm{dir})}+A_{i l}^{+} H_{j l}^{(\mathrm{dir})}-\frac{2}{3} A_{l m}^{+} H_{l m}^{(\mathrm{dir})} \delta_{i j}\right) \\
& -\frac{2}{7}\left(A_{i l}^{+} \frac{\partial}{\partial k}\left(k E H_{l j}^{(\mathrm{pol})}\right)+A_{j l}^{+} \frac{\partial}{\partial k}\left(k E H_{l i}^{(\mathrm{pol})}\right)-\frac{2}{3} \delta_{i j} A_{l m}^{+} \frac{\partial}{\partial k}\left(k E H_{l m}^{(\mathrm{pol})}\right)\right) \\
+ & \frac{1}{7} E\left(A_{l j}^{+} H_{i l}^{(\mathrm{pol})}+A_{l i}^{+} H_{j l}^{(\mathrm{pol})}-\frac{2}{3} A_{l m}^{+} H_{l m}^{(\mathrm{pol})} \delta_{i j}\right)-\frac{1}{3} E\left(A_{j l}^{-} H_{l i}^{(\mathrm{pol})}+A_{i l}^{-} H_{l j}^{(\mathrm{pol})}\right) .
\end{aligned}
$$

\section{Appendix 2. Explicit quadratic contributions of anisotropy for non-linear transfers}

Here are the complete expressions of the quadratic anisotropic contributions in the non-linear transfers for the anisotropic EDQNM modelling with truncation at the second order (MCS). The following convenient notation is used

$$
H_{l i}^{(\mathrm{dir})^{\prime \prime}} H_{l j}^{(\mathrm{dir})^{\prime}}+H_{l j}^{(\mathrm{dir})^{\prime \prime}} H_{l i}^{(\mathrm{dir})^{\prime}}-\frac{2}{3} H_{l n}^{(\mathrm{dir})^{\prime \prime}} H_{l n}^{(\mathrm{dir})^{\prime}} \delta_{i j}=\left\{H^{(\mathrm{dir})^{\prime \prime}}, H^{(\mathrm{dir})^{\prime}}\right\}_{i j} .
$$

The quadratic anisotropic isotropic transfer term is conservative and reads

$$
\begin{aligned}
Q^{\mathrm{NL}(\mathrm{iso})}(k, t)= & 20 \int_{\Delta_{k}} \theta_{k p q} \pi^{2} k^{2} p^{2} q \mathcal{E}_{0}^{\prime \prime}\left[2 x y\left(1-z^{2}\right) H_{l n}^{(\mathrm{pol})^{\prime \prime}}\left(\mathcal{E}_{0}^{\prime} H_{l n}^{(\mathrm{pol})^{\prime}}-\mathcal{E}_{0} H_{l n}^{(\mathrm{pol})}\right)\right. \\
& +6\left(x y+z^{3}\right)\left(2 H_{l n}^{(\mathrm{dir})^{\prime \prime}}\left(\left(3 x^{2}-1\right) \mathcal{E}_{0}^{\prime} H_{l n}^{(\mathrm{dir})^{\prime}}-\left(3 y^{2}-1\right) \mathcal{E}_{0} H_{l n}^{(\mathrm{dir})}\right)\right. \\
& \left.-H_{l n}^{(\mathrm{pol})^{\prime \prime}}\left(\left(1-x^{2}\right) \mathcal{E}_{0}^{\prime} H_{l n}^{(\mathrm{dir})^{\prime}}-\left(1-y^{2}\right) \mathcal{E}_{0} H_{l n}^{(\mathrm{dir})}\right)\right) \\
& +z\left(z^{2}-1\right)\left(H_{l n}^{(\mathrm{pol})^{\prime \prime}}\left(\left(1+x^{2}\right) \mathcal{E}_{0}^{\prime} H_{l n}^{(\mathrm{pol})^{\prime}}-\left(1+y^{2}\right) \mathcal{E}_{0} H_{l n}^{(\mathrm{pol})}\right)\right. \\
& \left.\left.-6 H_{l n}^{(\mathrm{dir})^{\prime \prime}}\left(\left(1-x^{2}\right) \mathcal{E}_{0}^{\prime} H_{l n}^{(\mathrm{pol})^{\prime}}-\left(1-y^{2}\right) \mathcal{E}_{0} H_{l n}^{(\mathrm{pol})}\right)\right)\right] \mathrm{d} p \mathrm{~d} q .
\end{aligned}
$$


The quadratic anisotropic directional transfer term is

$$
\begin{aligned}
Q_{i j}^{\mathrm{NL}(\mathrm{dir})}(k, t)= & \frac{20}{7} \int_{\Delta_{k}} \theta_{k p q} \pi^{2} k^{2} p^{2} q \mathcal{E}_{0}^{\prime \prime}\left[1 2 ( x y + z ^ { 3 } ) \left[(1+3 x y z) \mathcal{E}_{0}^{\prime}\left\{H^{(\mathrm{dir})^{\prime \prime}}, H^{(\mathrm{dir})^{\prime}}\right\}_{i j}\right.\right. \\
& \left.+\left(3 y^{2}-1\right) \mathcal{E}_{0}\left\{H^{(\mathrm{dir})^{\prime \prime}}, H^{(\mathrm{dir})}\right\}_{i j}\right]-6\left(x y+z^{3}\right)\left[\left(1-2 z^{2}-x y z\right) \mathcal{E}_{0}^{\prime}\left\{H^{(\mathrm{pol})^{\prime \prime}}, H^{(\mathrm{dir})^{\prime}}\right\}_{i j}\right. \\
& \left.+\left(1-y^{2}\right) \mathcal{E}_{0}\left\{H^{(\mathrm{pol})^{\prime \prime}}, H^{(\mathrm{dir})}\right\}_{i j}\right]+6 z\left(1-z^{2}\right)\left[\left(1-2 y^{2}-x y z\right) \mathcal{E}_{0}^{\prime}\left\{H^{(\mathrm{dir})^{\prime \prime}}, H^{(\mathrm{pol})^{\prime}}\right\}_{i j}\right. \\
& \left.-\left(1-y^{2}\right) \mathcal{E}_{0}\left\{H^{(\mathrm{dir})^{\prime \prime}}, H^{(\mathrm{pol})}\right\}_{i j}\right]+z\left(z^{2}-1\right)\left[\left(1-2 x^{2}-3 x y z\right) \mathcal{E}_{0}^{\prime}\left\{H^{(\mathrm{pol})^{\prime \prime}}, H^{(\mathrm{pol})^{\prime}}\right\}_{i j}\right. \\
& \left.-\left(1+y^{2}\right) \mathcal{E}_{0}\left\{H^{(\mathrm{pol})^{\prime \prime}}, H^{(\mathrm{pol})}\right\}_{i j}\right]-y\left(1-z^{2}\right)\left[(x+3 y z) \mathcal{E}_{0}^{\prime}\left\{H^{(\mathrm{pol})^{\prime \prime}}, H^{(\mathrm{pol})^{\prime}}\right\}_{i j}\right. \\
& \left.\left.+2 x \mathcal{E}_{0}\left\{H^{(\mathrm{pol})^{\prime \prime}}, H^{(\mathrm{pol})}\right\}_{i j}\right]\right] \mathrm{d} p \mathrm{~d} q .
\end{aligned}
$$

The quadratic anisotropic polarisation transfer term is

$$
\begin{aligned}
Q_{i j}^{\mathrm{NL}(\mathrm{pol})}(k, t)= & \frac{60}{7} \int_{\Delta_{k}} \theta_{k p q} \pi^{2} k^{2} p^{2} q \mathcal{E}_{0}^{\prime \prime}\left[-\left(x y+z^{3}\right)\left[2\left(3 x y z+2 z^{2}-1\right) \mathcal{E}_{0}^{\prime}\left\{H^{(\mathrm{dir})^{\prime \prime}}, H^{(\mathrm{pol})^{\prime}}\right\}_{i j}\right.\right. \\
& \left.+(1+x y z) \mathcal{E}_{0}^{\prime}\left\{H^{(\mathrm{pol})^{\prime \prime}}, H^{(\mathrm{pol})^{\prime}}\right\}_{i j}\right]+2\left(x y+z^{3}\right)\left[2\left(1-3 y^{2}\right) \mathcal{E}_{0}\left\{H^{(\mathrm{dir})^{\prime \prime}}, H^{(\mathrm{pol})}\right\}_{i j}\right. \\
& \left.+\left(1-y^{2}\right) \mathcal{E}_{0}\left\{H^{(\mathrm{pol})^{\prime \prime}}, H^{(\mathrm{pol})}\right\}_{i j}\right]-2 z\left(1-z^{2}\right)\left[6\left(x y z+2 x^{2}-1\right) \mathcal{E}_{0}^{\prime}\left\{H^{(\mathrm{dir})^{\prime \prime}}, H^{(\mathrm{dir})^{\prime}}\right\}_{i j}\right. \\
& -\left(3 x y z+2 y^{2}-1\right) \mathcal{E}_{0}^{\prime}\left\{H^{(\mathrm{pol})^{\prime \prime}}, H^{(\mathrm{dir})^{\prime}}\right\}_{i j}+6\left(1-y^{2}\right) \mathcal{E}_{0}\left\{H^{(\mathrm{dir})^{\prime \prime}}, H^{(\mathrm{dir})}\right\}_{i j} \\
& \left.-\left(1+y^{2}\right) \mathcal{E}_{0}\left\{H^{(\mathrm{pol})^{\prime \prime}}, H^{(\mathrm{dir})}\right\}_{i j}\right]+\left(y^{2}-z^{2}\right)\left[(x y+z) \mathcal{E}_{0}^{\prime}\left\{H^{(\mathrm{pol})^{\prime \prime}}, H^{(\mathrm{pol})^{\prime}}\right\}_{i j}\right. \\
& \left.+2(3 x y+z) \mathcal{E}_{0}^{\prime}\left\{H^{(\mathrm{dir})^{\prime \prime}}, H^{(\mathrm{pol})^{\prime}}\right\}_{i j}\right]-y\left(1-z^{2}\right)\left[4 x \mathcal{E}_{0}\left\{H^{(\mathrm{pol})^{\prime \prime}}, H^{(\mathrm{dir})}\right\}_{i j}\right. \\
& \left.\left.-(x+y z) \mathcal{E}_{0}^{\prime}\left\{H^{(\mathrm{pol})^{\prime \prime}}, H^{(\mathrm{pol})^{\prime}}\right\}_{i j}\right]\right] \mathrm{d} p \mathrm{~d} q .
\end{aligned}
$$

The quadratic anisotropic RTI term is

$$
\begin{aligned}
& Q_{i j}^{\mathrm{NL}(\mathrm{RTI})}(k, t)=\frac{120}{7} \int_{\Delta_{k}} \theta_{k p q} \pi^{2} k p^{2} q \mathcal{E}_{0}^{\prime \prime}\left[2 y(x+y z)(x y+z)(z k-q x) \mathcal{E}_{0}\right. \\
& \times\left(6\left\{H^{(\mathrm{dir})^{\prime \prime}}, H^{(\mathrm{dir})}\right\}_{i j}-6\left\{H^{(\mathrm{dir})^{\prime \prime}}, H^{(\mathrm{pol})}\right\}_{i j}+\left\{H^{(\mathrm{pol})^{\prime \prime}}, H^{(\mathrm{dir})}\right\}_{i j}-\left\{H^{(\mathrm{pol})^{\prime \prime}}, H^{(\mathrm{pol})}\right\}_{i j}\right) \\
& -k z(x y+z) \mathcal{E}_{0}^{\prime}\left(12\left(y(x+y z)-z\left(1-z^{2}\right)\right)\left\{H^{(\mathrm{dir})^{\prime \prime}}, H^{(\mathrm{dir})^{\prime}}\right\}_{i j}\right. \\
& -\left(6 y(x+y z)+2 z\left(1-z^{2}\right)\right)\left\{H^{(\mathrm{dir})^{\prime \prime}}, H^{(\mathrm{pol})^{\prime}}\right\}_{i j} \\
& +\left(2 y(x+y z)+6 z\left(1-z^{2}\right)\right)\left\{H^{(\mathrm{pol})^{\prime \prime}}, H^{(\mathrm{dir})^{\prime}}\right\}_{i j} \\
& \left.+\left(z\left(1-z^{2}\right)-y(x+y z)\right)\left\{H^{(\mathrm{pol})^{\prime \prime}}, H^{(\mathrm{pol})^{\prime}}\right\}_{i j}\right)+k(x y+z) \mathcal{E}_{0}^{\prime} \\
& \times\left(\left(6 y(y+x z)-4\left(1-z^{2}\right)\right)\left\{H^{(\mathrm{dir})^{\prime \prime}}, H^{(\mathrm{pol})^{\prime}}\right\}_{i j}\right. \\
& \left.\left.+y(y+x z)\left\{H^{(\mathrm{pol})^{\prime \prime}}, H^{(\mathrm{pol})^{\prime}}\right\}_{i j}\right)\right] \mathrm{d} p \mathrm{~d} q \text {. }
\end{aligned}
$$

